\title{
The census of cataclysmic variables in the ROSAT Bright Survey ${ }^{\star}$
}

\author{
A. D. Schwope ${ }^{1}$, H. Brunner ${ }^{1,3}$, D. Buckley ${ }^{2}$, J. Greiner ${ }^{1,3}$, K. v.d. Heyden ${ }^{2,4}$, S. Neizvestny ${ }^{5}$, \\ S. Potter ${ }^{2}$, and R. Schwarz ${ }^{1,6 \star \star}$
}

1 Astrophysikalisches Institut Potsdam, An der Sternwarte 16, 14482 Potsdam, Germany

2 South African Astronomical Observatory, PO Box 9, Observatory 7935, Cape Town, RSA

3 Max-Planck Institut für extraterrestrische Physik, Giessenbachstrasse, 85740 Garching, Germany

${ }^{4}$ SRON, Sorbonnelaan 2, 3584 CA Utrecht, The Netherlands

5 SAO RAS, Nizhnij Arkhyz, Zelenchukskaya, Karachaevo-Cherkesia, Russia, 357147

${ }^{6}$ Universitäts-Sternwarte Göttingen, Geismarlandstr. 11, 37083 Göttingen, Germany

Received 7 August 2002 / Accepted 19 September 2002

\begin{abstract}
We give an identification summary and results of polarimetric, photometric and spectroscopic follow-up observations of new, X-ray bright cataclysmic variables. These were identified as optical counterparts of high galactic latitude sources in the ROSAT All-Sky Survey. This optical identification programme is termed the ROSAT Bright Survey (RBS) and represents the first complete soft X-ray selected, flux-limited sample of CVs at high galactic latitude (survey area $\sim 20400$ sq.deg.). The systems described here escaped previous identification programmes since these surveys were designed to identify even brighter than ours or particularly soft X-ray sources. Among the 11 new RBS-CVs we find 6 magnetic systems of AM Herculis type, 4 dwarf novae (among them one candidate), and one particularly bright system of uncertain nature, tentatively identified as dwarf nova or symbiotic binary. Orbital periods could be determined for all magnetic systems which range from $87.1 \mathrm{~min}$ to $187.7 \mathrm{~min}$. Three of the new dwarf novae have moderate to high inclination and two of them might be eclipsing. Using non-magnetic systems only we derive a space density of $\mathrm{CVs}$ of $\sim 3 \times 10^{-5} \mathrm{pc}^{-3}$. This limit rests on the two new nearby, low-luminosity systems RBS0490 and RBS1955, with estimated distances of $30 \mathrm{pc}$ only and luminosities below $10^{30} \mathrm{erg} \mathrm{s}^{-1}$.
\end{abstract}

Key words. Surveys - X-rays: binaries - stars: cataclysmic variables

\section{Introduction}

In the ROSAT Bright Survey (RBS) we have completely optically identified all high-galactic latitude sources detected in the ROSAT All-Sky Survey with mean count rate above $0.2 \mathrm{~s}^{-1}$ (Schwope et al. 2000). About one third of all sources are of galactic origin. Most of them are stars with active coronae. Minority populations among the galactic constituents are interacting binaries of different flavour (neutron star accretors, white dwarf accretors, magnetic and non-magnetic systems, super-soft sources SSSs). With a survey area of more than 20000 square degrees and its flux limit of about $2.5 \times$ $10^{-12} \mathrm{erg} \mathrm{cm}^{-2} \mathrm{~s}^{-1}$ the RBS represents the deepest complete, soft X-ray selected, flux-limited sample of CVs (and other types of X-ray emitters) at high galactic latitude, important for population statistics and evolution scenarios of various kinds of X-ray emitters (see e.g. Miyaji et al. 2001 for the X-ray

Send offprint requests to: A. D. Schwope, e-mail: aschwope@aip.de

* Based in part on observations performed at the Eurpean Southern Observatory, La Silla, Chile, under programmes 60.B-0107 and 66.A-0664.

$\star \star$ Visiting astronomer, German-Spanish Astronomical Center, Calar Alto, operated by the Max-Planck-Institut für Astronomie, Heidelberg, jointly with the Spanish National Commission for Astronomy. luminosity function of AGNs based on the RBS and other Xray surveys).

Due to the soft spectral band of the PSPC detector, ROSAT identifications revealed a large number of new magnetic CVs, most of AM Herculis subtype (polars), and the SSSs as a new class of X-ray emitters. Polars are strongly magnetic $\mathrm{CV}$ where the magnetic field keeps both stars (in most cases) in synchronous rotation, inhibits the formation of an accretion disk and leads to intensive cyclotron radiation in the optical and $\mathrm{X}$-ray radiation from the accretion hot spot on the white dwarf. Compared to pre-ROSAT times with less than 20 systems, the number of polars has increased to almost 70, most of them identified as counterparts of very soft RASS or WFC-sources (Pye et al. 1995; Thomas et al. 1998; Beuermann et al. 1999). The discovery of new systems and the determination of their basic parameters (orbital period, masses) is of importance for the evolution of this kind of binary. The number density of the $\mathrm{CVs}$ in general is still highly uncertain. Their observed minimum period is far from being compatible with current theoretical models and the presence or absence of the 2-3 hour period gap and the likely causes for its presence or absence are a matter of debate.

However the restriction to soft X-ray sources a priori excludes the vast majority of CVs to be detected: normal disk systems in quiescence which have intrinsically hard spectra. 
Table 1. X-ray and optical summary information about new RBS-CVs. Listed are the RBS catalog number, the RASS catalog number containing the X-ray coordinates, the RASS count rate and hardness ratio HR1, the coordinates of the optical counterpart, the magnitude in the optical range, the orbital period (if known), the X-ray to optical flux ratio and the suggested sub-class of interacting binary. The typical magnitude uncertainty is $0.1-0.2 \mathrm{mag}$.

\begin{tabular}{|c|c|c|c|c|c|c|c|c|}
\hline RBS-No & 1RXS J & $\begin{array}{l}\mathrm{CR} \\
{\left[\mathrm{s}^{-1}\right]}\end{array}$ & $\begin{array}{c}\text { HR1 } \\
\pm\end{array}$ & RA(2000) & $\overline{\mathrm{DEC}(2000)}$ & $\begin{array}{c}m_{V} \\
{[\mathrm{mag}]}\end{array}$ & $\begin{array}{c}P_{\text {orb }} \\
{[\mathrm{min}]}\end{array}$ & Type \\
\hline 206 & $012851.9-233931$ & 0.34 & $-0.84 \pm 0.04$ & $01: 28: 52.3$ & $-23: 39: 44$ & $17^{\mathrm{m}} \cdot 5-18.0^{\mathrm{m}} 0^{(a)} / 18^{\mathrm{m}} \cdot 9^{(b)}$ & 92.8 & $\mathrm{AM}$ \\
\hline 324 & $023052.9-684203$ & 0.26 & $-0.70 \pm 0.09$ & 02:30:50.9 & $-68: 42: 06$ & $16.0-18.0^{(a)}$ & 181.8 & $\mathrm{AM}$ \\
\hline 372 & $025538.2-224655$ & 0.23 & $-0.11 \pm 0.13$ & $02: 55: 37.9$ & $-22: 47: 02$ & 17.8 & - & DN \\
\hline 490 & 035410.4-165244 & 0.23 & $+0.57 \pm 0.08$ & $03: 54: 10.3$ & $-16: 52: 45$ & $16^{\mathrm{m}} 0$ & - & DN \\
\hline 541 & $042555.8-194534$ & 0.23 & $+0.18 \pm 0.12$ & $04: 25: 55.2$ & $-19: 45: 30$ & $16^{\mathrm{m}} \cdot 9$ & 87.1 & $\mathrm{AM}$ \\
\hline 696 & $082051.2+493433$ & 0.25 & $-0.93 \pm 0.03$ & $08: 20: 50.9$ & $+49: 34: 31$ & $18 \cdot 0-19 \cdot 3^{(a)}$ & 99.4 & $\mathrm{AM}$ \\
\hline 1411 & $143703.5+234236$ & 0.25 & $+0.57 \pm 0.08$ & $14: 37: 03.4$ & $+23: 42: 27$ & $19 \mathrm{~m} \cdot 1$ & - & $\mathrm{DN}$ \\
\hline 1563 & $161008.0+035222$ & 0.54 & $-0.41 \pm 0.11$ & $16: 10: 07.5$ & $+03: 52: 33$ & $15^{\mathrm{m}} \mathrm{9}$ & 187.7 & $\mathrm{AM}$ \\
\hline 1735 & $211540.9-584045$ & 0.38 & $-0.02 \pm 0.10$ & $21: 15: 40.6$ & $-58: 40: 52$ & $17 \mathrm{~m} .8$ & 110.8 & $\mathrm{AM}$ \\
\hline 1955 & $230949.6+213523$ & 0.21 & $+0.20 \pm 0.10$ & 23:09:49.2 & $+21: 35: 19$ & $15^{\mathrm{m}} \cdot 6$ & - & DN/Symb. \\
\hline 1969 & $231532.3-304855$ & 0.28 & $+0.31 \pm 0.22$ & $23: 15: 31.7$ & $-30: 48: 48$ & $16^{\mathrm{m}} \cdot 6$ & $84.2^{(c)}$ & DN \\
\hline
\end{tabular}

\footnotetext{
(a) Orbital high state variability.

(b) Low state magnitude.

${ }^{(c)}$ Chen et al. (2001).
}

Population synthesis using standard CV evolution predicts a space density of $\sim 10^{-5} \mathrm{pc}^{-3}$ (de Kool 1992) to $\sim 10^{-4} \mathrm{pc}^{-3}$ (Politano 1996), with the consequence that about 10 to 100 undetected CVs should be hiding in the local vicinity $(50 \mathrm{pc})$ of the Sun. Altough most of these sources have been evolved to short periods, and accrete only at low $\dot{M}$, they should still emit $L_{\mathrm{X}} \sim 10^{30} \mathrm{erg} \mathrm{s}^{-1}$ and therefore show up in any medium deep X-ray survey. The only pre-ROSAT medium deep X-ray search for CVs, the Einstein galactic plane survey (Hertz et al. 1990) detected 3 faint disk CVs and one AM Herculis system in a 144 sqr degree field. The implied space density was $(2-3) \times 10^{-5} \mathrm{pc}^{-3}$ in agreement with the theoretical predictions, but a factor of 5 to 10 higher than estimates based other detection criteria (variability, spectra, color) (see e.g Patterson 1984).

DQ Herculis stars or intermediate polars are another class of CVs which are X-ray bright. They possess significant magnetic fields but the magnetic moment is too weak to synchronize both stars. Mass transfer may include an accretion disk and the systems have in general hard X-ray spectra. Most DQ Herculis stars have periods above 3 hours and it's not clear whether they later evolve into AM Herculis stars or form a largely unrecognized population. Thus an unbiased sample of $\mathrm{CVs}$ is crucial to validate our current understanding of their evolution.

For several of the new CVs found in the RBS (Schwope et al. 2000) follow-up observations were initiated. In this paper we present the current status of the observations of these systems, including the results of photometric, polarimetric and spectroscopic observations. Results of our initial studies of RBS0206 and RBS1735, now called EQ Cet und CD Ind, respectively, were presented earlier (Schwope et al. 1997, 1999). The main properties of the objects under investigation in this paper are summarized in Table 1.

The paper is organized in the following way. We briefly describe our optical observations and then present the results and implications in sections devoted to individual objects, separating magnetic and non-magnetic systems. The paper is closed with a short discussion of all CVs in the RBS.

\section{Observational summary of new interacting binaries found in the RBS}

The spectra shown in this paper were obtained as part of the identification program of all RBS-sources (Schwope et al. 2000) using the 6m-telescope at Zelenchukskaya (equipped with the UAGS $+\mathrm{CCD}$ prime focus spectrograph), the ESO $3.6 \mathrm{~m}$-telescope equipped with EFOSC2, the Calar Alto $3.5 \mathrm{~m}$ telescope with MOSCA, and the ESO $1.5 \mathrm{~m}$ telescope equipped with a Boller \& Chivens spectrograph. Low-resolution grisms or gratings were used in all cases, which gave spectra covering the whole optical wavelength range with moderate to low resolution (8-20 $\mathrm{A} F W H M$ ). Follow-up spectroscopy, partly with higher resolution (4 $\AA F W H M)$ was obtained with the SAAO $1.9 \mathrm{~m}$ telescope on several occasions in 1998 and 1999.

Follow-up photometry and polarimetry was performed with the ESO/Dutch $90 \mathrm{~cm}$ telescope with CCD camera, the AIP $70 \mathrm{~cm}$ telescope with CCD camera, and the SAAO $1.9 \mathrm{~m}$ telescope with the UCT polarimeter. More detailed information about the observations is given in the sections on the individual targets.

Finding charts of the new CVs are reproduced in the Appendix. All charts have a size of $5 \times 5$ arcmin, North at the top and East to the left. The CVs are marked by short dashes.

\section{New magnetic cataclysmic variables}

\section{1. $R B S 0206$ (= 1RXS J012851.9-233931)}

The identification of RBS0206 as a magnetic cataclysmic variable of AM Herculis type was announced by Schwope et al. (1999). The identification was based on one low-resolution spectrum taken in a low accretion state and some occasional 


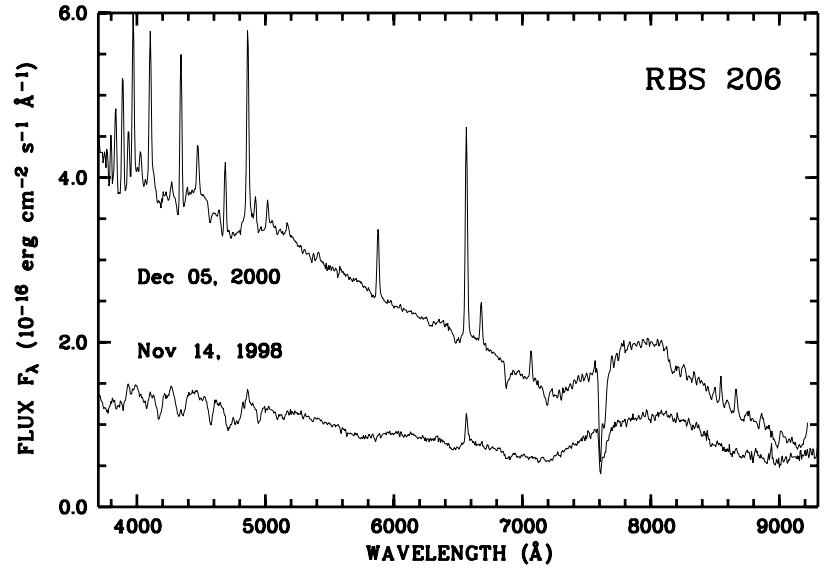

Fig. 1. Single low-state discovery spectrum and mean high-state spectrum of RBS0206 taken during follow-up observations with the ESO $3.6 \mathrm{~m}$ telescope. Observation dates are indicated in the figure.

I-band photometry. The spectrum showed one pronounced cyclotron line at $\sim 8000 \AA$ probably originating in a low temperature accretion plasma, $k T \sim 2 \mathrm{keV}$, with a field strength of $45 \mathrm{MG}$, and Zeeman shifted photospheric Balmer absorption lines indicating a mean magnetic field strength of $\sim 36 \mathrm{MG}$. The photometry suggested a periodicity of about 90 or $146 \mathrm{~min}$, respectively.

Follow-up photometry, polarimetry, and low-resolution spectroscopy was obtained using the SAAO $1.9 \mathrm{~m}$ telescope equipped with the UCT polarimeter in September and December 2001, and with the ESO $3.6 \mathrm{~m}$ telescope equipped with EFOSC2 in December 2000. Results of the new observations are shown in Figs. 1 to 4.

In December 2000 a total of 9 spectra were taken with individual integration times of $900 \mathrm{~s}$. The spectra cover the wavelength range $3700-9300 \AA$ with a $F W H M$ resolution of about $20 \AA$. The mean spectrum of the follow-up observations is reproduced in Fig. 1 in comparison with the discovery spectrum taken with the same instrumental setup at the same telescope. In December 2000 the source has returned to a high accretion state which is evident from the strong emission lines of hydrogen, neutral and ionized helium, the disappearance of the photospheric absorption lines and the blue continuum. The low-state spectrum indicated a system brightness of $V \simeq 18$. 9 , in the high state of December 2000 the source varied between $V=17.5$ and 18.0

Despite the large differences between the low and high state spectra, there is one striking similarity, the pronounced cyclotron line at $8000 \AA$. This line is almost equally luminous in both accretion states which is indicative of a low-temperature accretion region persistently present irrespective of the mass accretion rate.

The photometric and polarimetric observations were used in order to derive the spin period of the white dwarf. Data were obtained in 5 nights between HJD 2452258 and 2452261 comprising about 1500 cycles of the binary. The light curves and circular polarization curves obtained in three of the nights are shown in Fig. 2. Filters and observation dates are indicated in

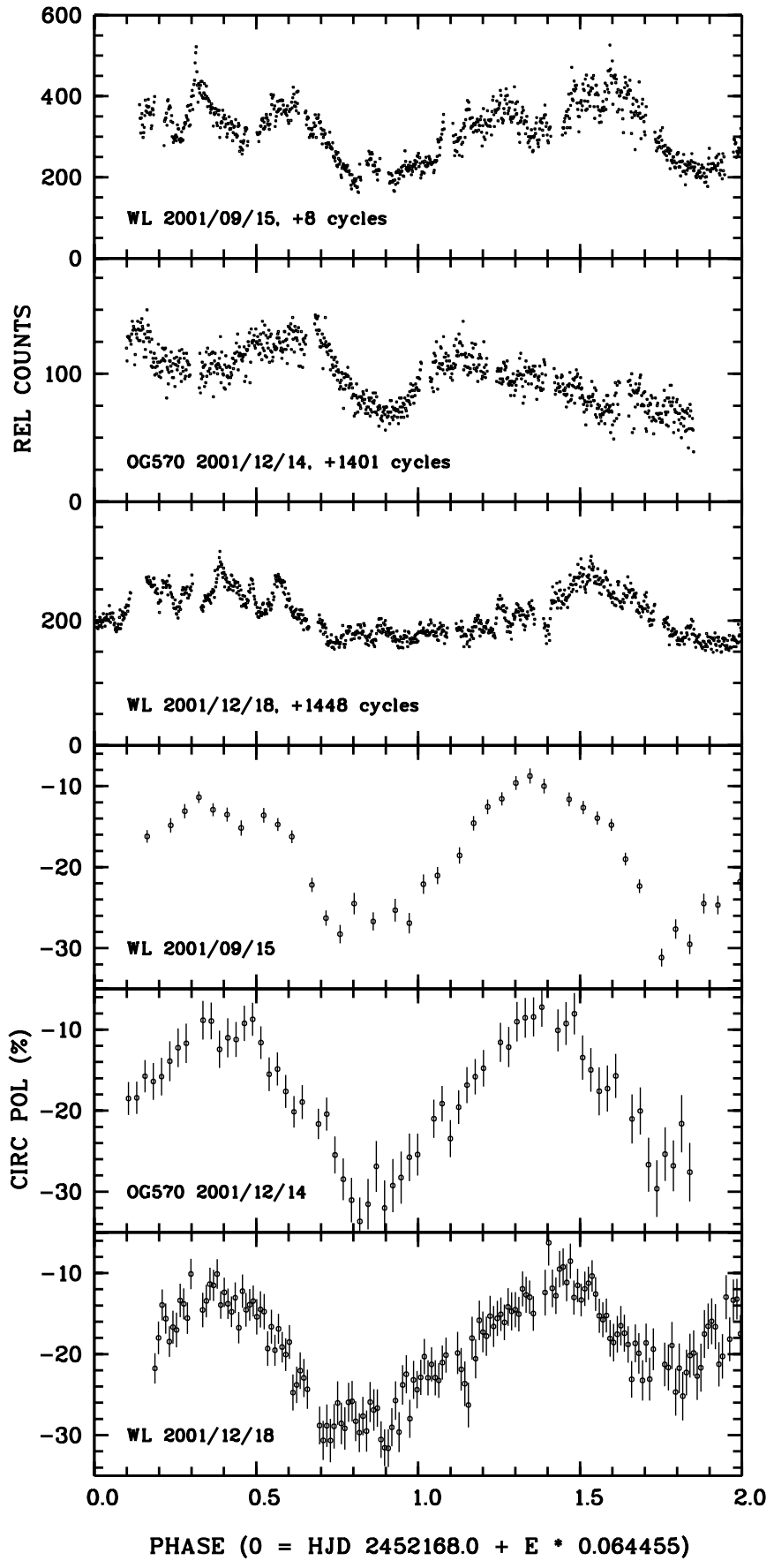

Fig. 2. Photometry and polarimetry of RBS0206 with the SAAO $1.9 \mathrm{~m}$ telescope. Dates and filters used are indicated in the individual panels. The top three panels show the photometry, the bottom panels the circular polarimetry. The data are phased according to the ephemeris at the abscissa, but shown in original time sequence.

the figure. Linear polarization observations were also obtained but revealed no clear detection of linearly polarized light.

Photometric variations by a factor of 2 are evident from the figure. There seems to be a kind of on-off pattern present in the light curves with a pronounced photometric minimum at phase 0.9 (in filter OG570), the photometric minimum and the length of the bright phase, however, are very unstable. 
The photometric variations did not yield reliable periods when period search algorithms were applied to these data.

The circular polarization signal displayed much smoother variation between $-10 \%$ and $-30 \%$ at any of the five occasions. The polarization curves were found to be much more stable than the light curves itself and were used for a period search with the analysis-of-variance method provided by the MIDAS data reduction package. A periodogram showed the most prominent peak at $f=15.5147$ cycles per day, corresponding to a period of $92.815 \pm 0.003 \mathrm{~min}$. This is accepted as the spin period of the white dwarf and likely to be the orbital period of the binary. Phases in Fig. 2 were calculated using this period with the zero point chosen arbitrarily at the beginning of the photometric campaign. The accuracy of the polarimetric period is of sufficient accuracy to connect the $\sim 1500$ cycles covered in 2001, but is insufficient to extend back to the earlier observations.

The high degree of the circular polarization is striking und underlines the magnetic nature of RBS0206. The nonvanishing circular polarization indicates that the accretion spot where the cyclotron radiation originates never rotates behind the limb of the white dwarf but is continously in view. The high degree of circular polarization at phase $0.8-0.9$ on the other hand suggests that we are almost looking pole-on at this phase. Both geometrical constraints together indicate $i \simeq \delta \simeq 40^{\circ}$ ( $i$ : orbital inclination, $\delta$ : latitude of the field line in the accretion spot with respect to the rotation axis).

The emission lines in the nine spectra obtained in December 2000 display radial velocity variations. When fitted with a sine curve, the $\mathrm{H} \alpha$ lines give a radial velocity amplitude of $125 \mathrm{~km} \mathrm{~s}^{-1}$ and a period of $96 \pm 4 \mathrm{~min}$. The latter value is compatible with the polarimetric period and thus suggestive of $P_{\text {spin }}=P_{\text {orb }}$. Maximum recessional velocity occurs at optical minimum. It is assumed, that the optical light curve is modulated mainly by the beaming properties of the cyclotron source. Optical minimum occurs, when the observer is looking poleon. The phasing of the radial velocity curve then implies that the bulk of the line radiation originates from the magnetically channeled accretion stream.

\subsubsection{Cyclotron spectroscopy of RBS0206}

The nine low-resolution spectra obtained in December 2000 were phase-folded using the $92.8 \mathrm{~min}$ spin period. This resulted in spectra at 6 independent phases. These are shown in Fig. $3 \mathrm{a}$ as $F_{\lambda}$ vs. $\lambda$. The two lowest spectra were obtained in the photometric minimum. They still show the strong cyclotron emission line at $8000 \AA$ found also in the low accretion state. Apart from this line the minimum spectra do not show further pronounced cyclotron humps.

The four spectra obtained in the bright phase, however, show a complete system of cyclotron humps at wavelengths shorter than $8000 \AA$. These lines show a strong shift in wavelength as a function of phase, whereas the line at $8000 \AA$ remains stationary. In the third spectrum from the top in Fig. $3 \mathrm{a}$ the humps are centered at $6700,5800,5000,4400$, and $4030 \AA$, respectively. This spectrum will be investigated in more detail below.

In order to underline the phase-dependent motion of the cyclotron lines we show the four bright phase spectra after subtraction of the average minimum spectrum in Fig. $3 \mathrm{~b}\left(F_{v}\right.$ vs. $\left.v\right)$ and connect two of the cyclotron harmonics with a curved line. The shift of the harmonics is of order of half the separation between two humps. It would be quite natural to assume, that the additional cyclotron humps at short wavelengths are higher harmonics of the same source of radiation which emits the pronounced line at $8000 \AA$. This interpretation has severe difficulties, since the $8000 \AA$ hump and those at shorter wavelengths cannot be explained with one common field strength. In addition, the $8000 \AA$ hump remains stationary, whereas the other lines move with phase by several hundreds of Ångstroms (see Fig. 3b).

We were thus forced to assume that the bright phase spectrum of RBS0206 is fed by two independent sources of radiation. Modeling of these two sources was performed for the bright-phase in the following manner. We estimated the contribution of the accretion stream to the optical spectrum by assuming that the difference spectrum between optical minimum in the high state and the low-state discovery spectrum is solely due to emission from the stream. This spectrum is shown as the lower curve in the upper panel of Fig. 4. The upper curve is the average of the two high-state spectra which show the most pronounced and most redshifted cyclotron lines $\left(2^{\text {nd }}\right.$ and $3^{\text {rd }}$ from top in Fig. 3). According to cyclotron theory this aspect corresponds to the maximum angle between the line of sight and the magnetic field in the emitting plasma. From polarimetry we estimated this angle to be of order $\Theta \simeq 80^{\circ}$ (see above). The difference between the two spectra in the upper panel of Fig. 4 is regarded as pure cyclotron radiation and shown in the lower panel of that figure.

We compare this spectrum with suitably scaled superpositions of homogeneous, isothermal cyclotron spectra. Since we are lacking sufficient phase resolution and phase coverage for a detailed comparison of the models with the observations, these shall be regarded as tentative only.

The model rising to the blue end of the spectrum was computed for $k T=10 \mathrm{keV}, \Theta=80^{\circ}, B=32 \mathrm{MG}$. We refer to this model as spot(2) in Fig. 3. It is a superposition of three spectra with different plasma (density) parameters $\Lambda$ and an optically thick Rayleigh-Jeans part. The value of $\Lambda$ varies between $10^{5}$ and $10^{8}$. This choice ensures that sufficient high harmonics remain optically thin in the individual spectra. A rather high temperature of $10 \mathrm{keV}$ was chosen for this part of the spectrum, since only at temperatures as high as this, do harmonics move with aspect angle i.e. spin phase (see Schwope 1990 for a general discussion of the motion of cyclotron humps). The general slope of the spectrum as well as the position of the cyclotron humps shortward of $7300 \AA$ are well described with this model. It fails to reproduce the pronounced hump at $8000 \AA$, both in position and intensity.

The dashed line in the lower panel of Fig. 4 is the same model as shown in the discovery paper by Schwope et al. (1999). It was computed for a temperature of $2 \mathrm{keV}, \Theta=50^{\circ}$, $\Lambda=10^{2}, B=45 \mathrm{MG}$, and could well reproduce the shape and 

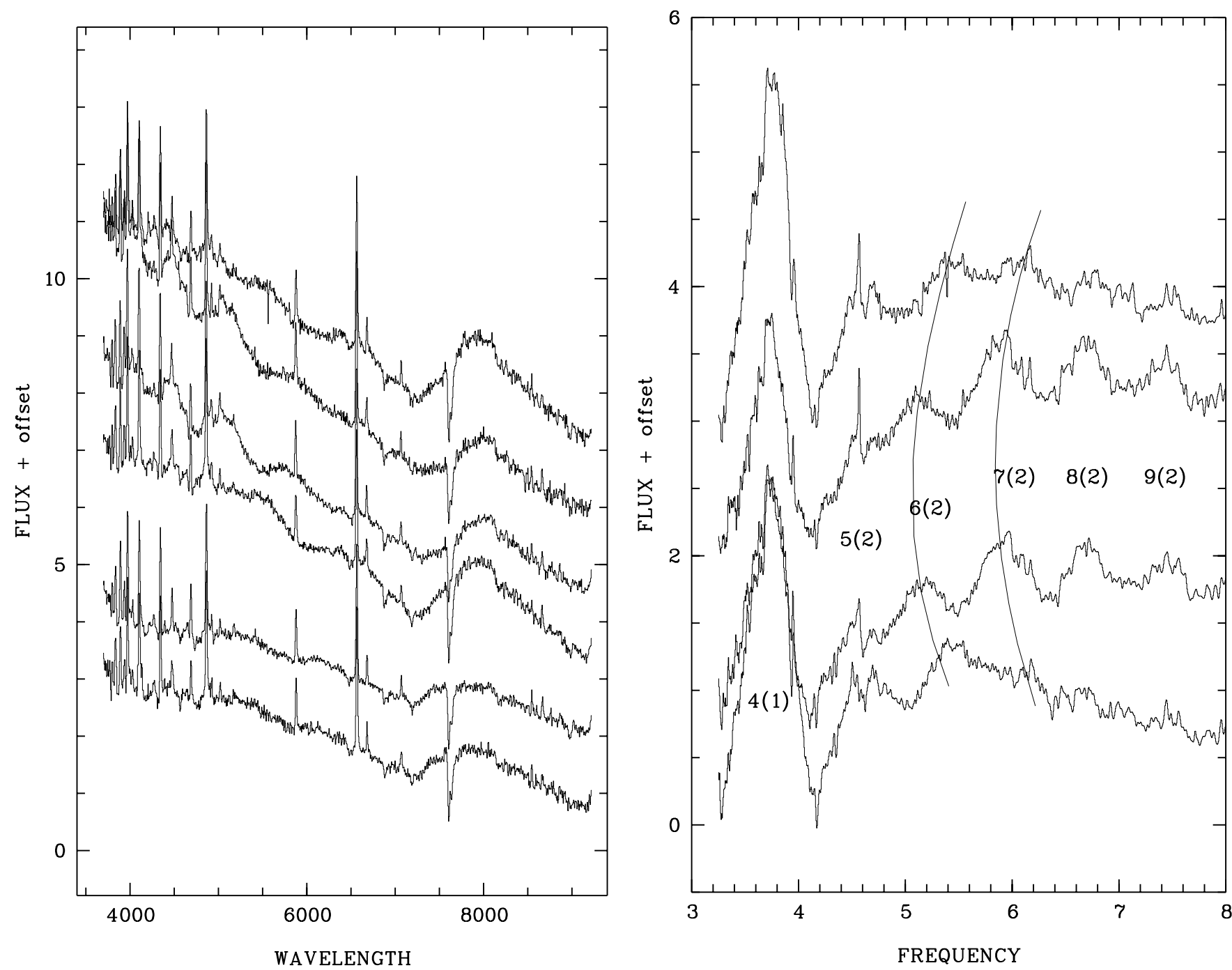

Fig. 3. left, a) Low-resolution spectra around the spin cycle of RBS0206. Flux units are $10^{-16} \mathrm{erg} \mathrm{cm}^{-2} \mathrm{~s}^{-1}$. The individual spectra were plotted with an offset of 1.3 flux units with respect to each other. right, b) The top four spectra of a) after subtraction of the minimum spectrum, represented as $F_{v}$ vs. $v$, plotted with an offset of 1 flux unit with respect to each other. Frequencies are given in $10^{14} \mathrm{~Hz}$. Individual cyclotron humps of the two accretion spots are labeled by integer harmonic numbers.

position of the hump at $8000 \AA$ A. Due to the very low value of $\Lambda$ it could also explain the non-detection of the next higher harmonic (the fourth), which was completely optically thin and just too weak to be detectable.

The presence of two accretion spots at the same time, one with $32 \mathrm{MG}$, the other with $45 \mathrm{MG}$, seems to be puzzling, as well as the extreme low density of the spot which emits the $8000 \AA$ hump. We therefore explored whether the hump at $8000 \AA$ A could also be explained with plasma emission in a field of strength of about $32 \mathrm{MG}$. A single low-temperature spectrum, $k T=2 \mathrm{keV}, B=33.7 \mathrm{MG}$, indeed reveals a hump at the observed wavelength, the fourth harmonic of the cyclotron fundamental (indicated as this in Fig. 3b). Such a line, however, is much too narrow in order to explain the cyclotron line as a whole.

We therefore calculated a superposition of lowtemperature, low-density, $k T=2 \mathrm{keV}, \log \Lambda=1$, cyclotron models for a range of field strengths between 32 and 35.5 MG. Such a model is shown as dotted line in the lower panel of
Fig. 4 and it reproduces the $8000 \AA$ hump very well. At the given low temperature, both models ( 33 and $45 \mathrm{MG}$ ) predict no considerable motion of the cyclotron humps as a function of phase. We conclude that we cannot distinguish between the alternative models with the present data. The high-field model is attractive for its simplicity, the low-field model is attractive for the field strength being close to the value used for modeling of the blue part of the spectrum.

Both scenarios are possible, two spots at more or less the same location (field strength) on the white dwarf or two spots with some separation, at least as far as the field strength is concerned. One region is rather dilute and persistently accreting during low and high accretion states, the other region whose density is in the normal range for polars, is accreting in the high state only. There is no firm clue on the accretion geometry apart from the likely combination $i \simeq \delta \simeq 40^{\circ}$, which means that both accretion spots are continously in view. In order to disentangle the accretion geometry one needs to locate the secondary star in the orbit. Since the companion is not seen 


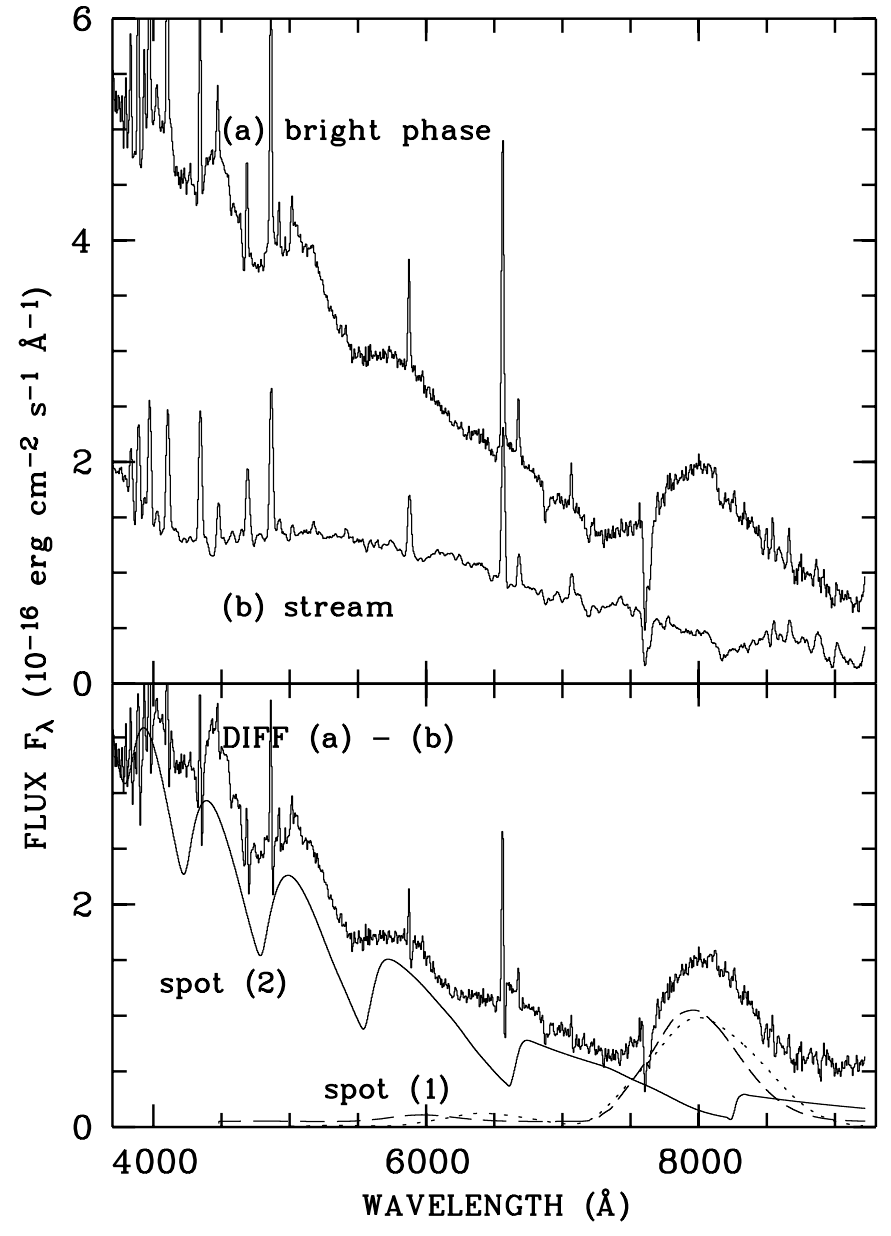

Fig. 4. In the upper panel a high-state, bright-phase spectrum of RBS0206 is shown. The spectrum below is the difference between the orbital minimum in the high state and the single low-state spectrum obtained in the 1998 low state. In the lower panel the difference spectrum is shown, which is regarded as of pure cyclotron origin. The three cyclotron models are explained in the text.

directly, the only way to achieve this goes via the identification of an emission line of reprocessed origin from the surface of the secondary in a high accretion state. Further observations, both spectroscopically and polarimetrically, are highly demanded in order to unveil the secrets of this intriguing system.

\section{2. $R B S 0324(=1 R X S J 023052.9-684203)$}

The identification spectra of \#324, obtained with the ESO $1.5 \mathrm{~m}$ telescope, showed the typical line features of a high-accretion rate AM Herculis binary (polar), broad, asymmetric emission lines of $\mathrm{H}, \mathrm{HeI}$ and HeII. Follow-up photometric, polarimetric, and spectroscopic observations were performed with the SAAO $1.9 \mathrm{~m}$ telescope at several occasions (August 1998 - December 2001), and the ESO/Dutch $90 \mathrm{~cm}$ telescope (October 1998). These observations consistently revealed a period of $181.8 \mathrm{~min}$, which we regard as the binary period of the system. The results of polarimetric observations obtained in December 2001 are shown in Fig. 5. These data show the high degree of variability of the source on timescales of minutes and hours. In its high state, the source varied between $V=16^{\mathrm{m}}$ and $18^{\mathrm{m}}$. Polarimetry immediately proves the tentative identification as a polar through the high degree of circular polarization, reaching $20 \%$ at certain phases, modulated with the period of the binary.

For future use, e.g. the derivation of a long-term ephemeris, we list in Table 2 the times of the centroids of the bright phase as obtained by Gaussian fits to the light curves at the different occasions in 1998 and 2001.

Table 2. Times of the center of the bright phase of RBS0324.

\begin{tabular}{cl}
\hline \hline $\begin{array}{c}\text { Date } \\
\text { YYMMDD }\end{array}$ & $T_{\text {cent }}$ \\
[HJD] \\
\hline 981029 & 2451115.6722 \\
981031 & 2451117.5649 \\
981101 & 2451118.8266 \\
011219 & 2452263.3265 \\
011219 & 2452263.4543 \\
011223 & 2452267.3632 \\
\hline
\end{tabular}

The light curve and polarimetric variation can be naturally explained by strong cyclotron beaming of one accreting pole, which is continously in the observable hemisphere. At photometric maximum the angle between the observer and the magnetic field in the accretion spot is largest. At photometric minimum we are observing almost from above the spot. This should lead to a high degree of circular polarization at this phase. Indeed an increase of the degree of polarization is observed at beginning and end of the faint phase, in the center of the faint phase however the signal becomes depolarized. This is likely due to depolarization by background radiation (photospheric radiation from the stars and recombination radiation from the accretion stream) and possibly due to absorption in the intervening accretion stream. The pattern of photometric and polarimetric variability resembles to a large extent that of MR Ser (Liebert et al. 1982) and we propose a similar accretion geometry. The orbital inclination is about $i \simeq 40^{\circ}$, the co-latitude of the magnetic field $\delta$ in the accretion spot must be almost the same. Such a geometry ensures a large variation of the projection angle of the magnetic field in the spot, which in turn causes pronounced polarization variations. It also ensures, that the $i+\delta<90^{\circ}$, so that no circular polarization sign reversal occurs.

Low-resolution spectra at orbital minimum and orbital maximum are shown in Fig. 6. The difference is regarded as cyclotron spectrum from the accretion spot(s). The difference spectrum is a smooth function of the wavelength with maximum at $\sim 5500 \AA$. Individual cyclotron harmonics cannot be resolved, so that no direct clue to the magnetic field strength can be derived. The shape of the cyclotron spectrum is very similar to that of EF Eri (Schwope 1991), which has a high temperature $(k T \simeq 20 \mathrm{keV})$, optically thick (plasma parameter $\left.\Lambda \simeq 10^{8}\right)$, low-field accretion plasma $(B=13 \mathrm{MG})$. We can readily assume similar plasma parameters for RBS0324. Contrary to EF Eri, we cannot derive independent evidence for the low field in RBS0324 by Zeeman absorption lines, neither 

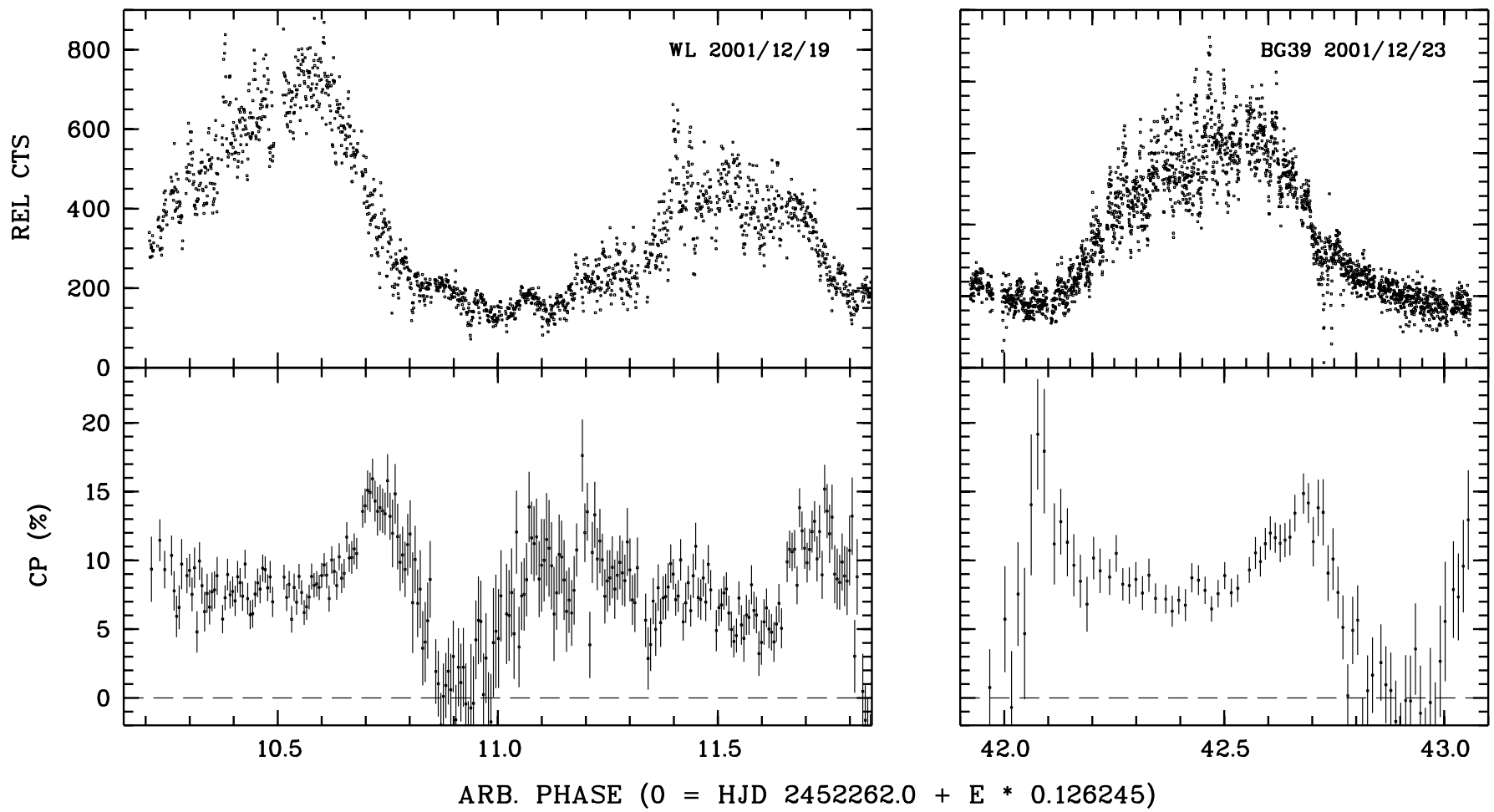

Fig. 5. Polarimetry of RBS0324 obtained December 2001 with the $1.9 \mathrm{~m}$ SAAO. Shown are relative brightness (top) and the degree of circular polarization. Data are shown in original sequence and were not phase-folded. The data on December 19 were taken in white light, those at December 23 through a blue BG39 filter.

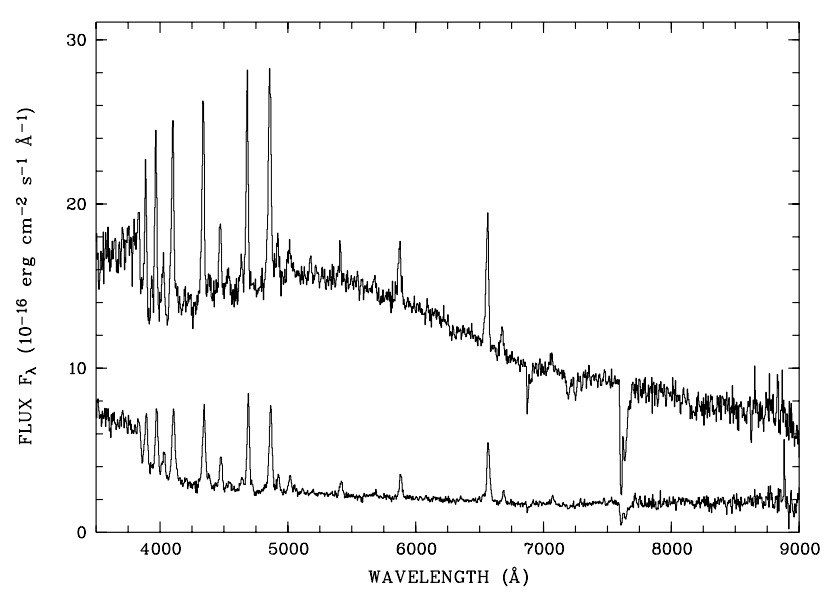

Fig. 6. Identification spectra of RBS0324, obtained with the ESO $1.5 \mathrm{~m}$ telescope on December 1997. The two spectra represent the orbital minimum and maximum, respectively.

from the accretion halo nor from the white dwarf's photosphere (Östreicher et al. 1990).

The spectra of Fig. 6 display TiO absorption bands from the secondary star in the near infrared. The low signal to noise ratio of the spectra prevents us from properly determining the spectral type. At the orbital period of \#324, a Roche-lobe filling dwarf star secondary has a spectral type dM4 (see e.g. the fit by Gänsicke et al. (1995) to the secondary in AM Her at the same orbital period). We determined the contribution of the secondary to the spectral hump at $7520 \AA$ to $20-25 \%$, which implies a distance to RBS0324 of about $d=250 \mathrm{pc}$.

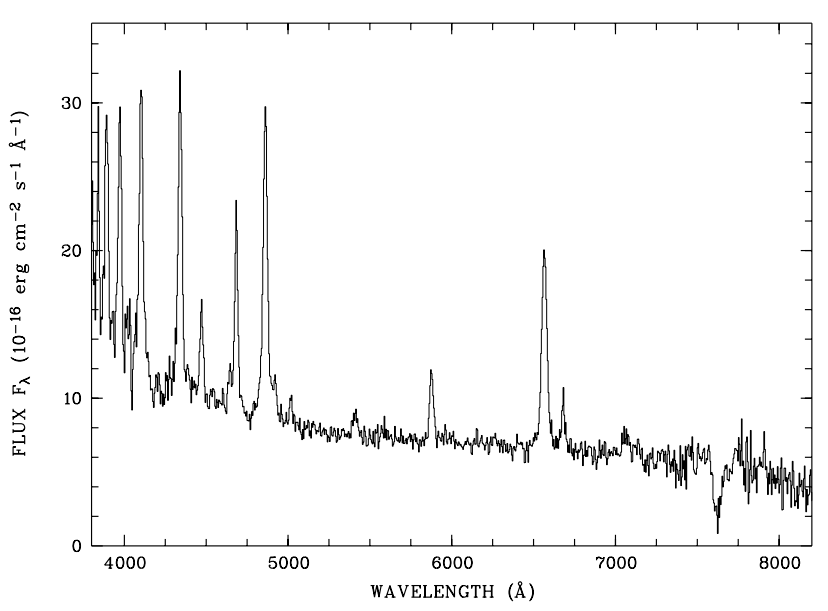

Fig. 7. Discovery spectrum of RBS0541, obtained October 7, 1996, at Zelenchukskaya.

The small number of 70 photons was detected during the RASS only. The RASS X-ray spectrum is very soft and compatible with one blackbody-like component only $(k T \simeq 30 \mathrm{eV})$. Such a fit is similar to the soft component of many AM Her stars (Beuermann \& Schwope 1994) including the prototype (Paerels et al. 1994).

\section{3. $R B S 0541$ (= $1 R X S J 042555.8-194534)$}

The low-resolution discovery spectrum of RBS0541, taken at Zelenchukskaya, shows a smooth blue continuum and 


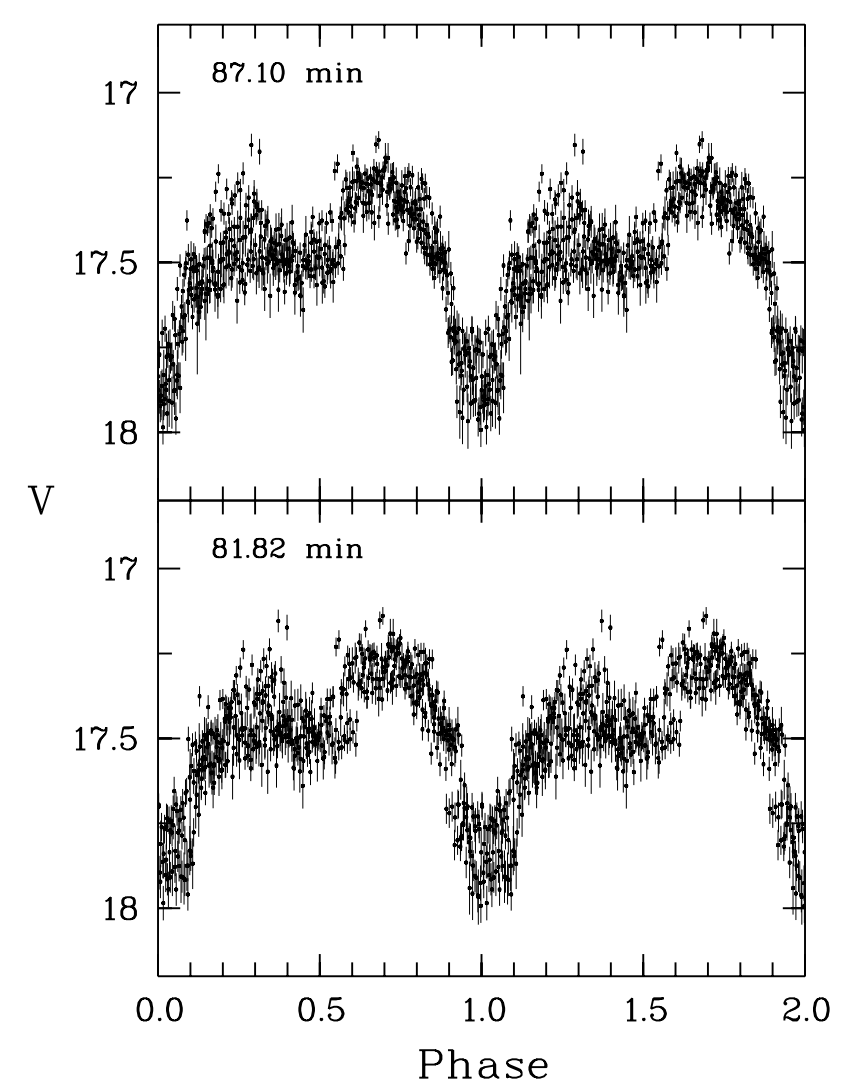

Fig. 8. CCD-photometry of RBS0541, folded over the possible alias periods (observation dates October 28-31, 1998). Shown are differential $V$-band magnitudes as a function of orbital phase. All data are shown twice for clarity.

asymmetric emission lines of $\mathrm{H}, \mathrm{HeI}$, and HeII. There is no obvious sign of the secondary star in the spectrum. The below-slit magnitude of \#541 at October 7, 1996, was $V=16.7$, similar to the brightness listed in the USNO-A2 catalogue. Attempts were made to obtain further spectra with the $1.9 \mathrm{~m}$ telescope at SAAO on August 20 and 23, 1998, and January 22, 1999. These attempts failed due to the faintness of the system at these occasions, indicating a deep low state of the binary.

Time-resolved CCD-photometry with the ESO/Dutch $90 \mathrm{~cm}$ telescope at La Silla was obtained in the nights Oct. 2831,1998 , through a $V$-filter. Source intensities were determined using the DoPhot package (Mateo \& Schechter 1989) and intensities were reduced to the brightness of the 14.77 comparison star USNO A2 U0675_01566006 at RA = 4:26:00.41, DEC $=-19: 45: 57.4$. The source showed an overall brightness decline by $\sim 0.4 \mathrm{mag}$ during those days. Apart from this, RBS0541 displayed pronounced periodic intensity variations with an amplitude of $\sim 0.8$ mag between $V \simeq 17.2$ and $\sim 18.0$ (Fig. 8). A period search with the analysis of variance technique implemented in MIDAS yielded a likely period of $87.1 \mathrm{~min}$ with a possible alias period of $81.8 \mathrm{~min}$. The folded, de-trended light curves for both possible periods are shown in Fig. 8. Times of optical minima in RBS0541 are 2451115.8014, 2451115.8608, 2451116.7691, 2451117.6761, and 2451118.6447.

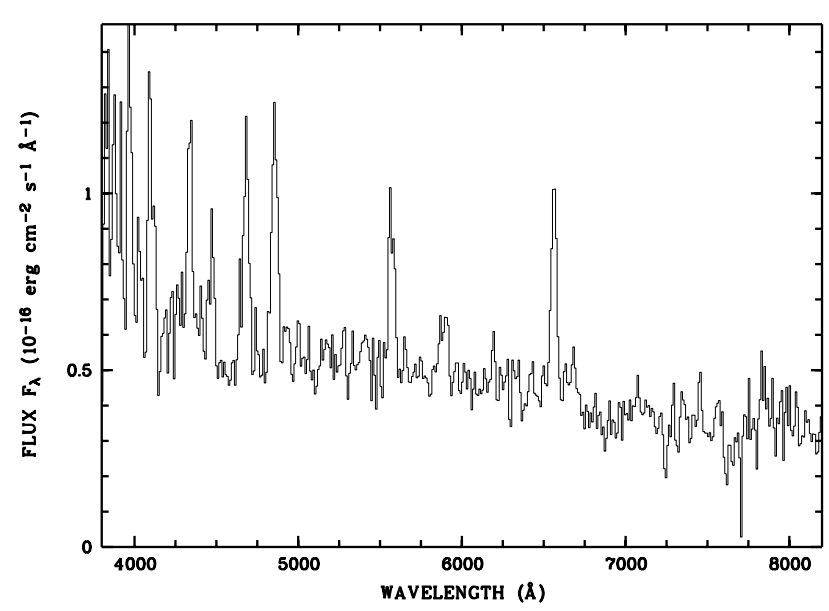

Fig. 9. Identification spectrum of RBS0696 taken with MOSCA at the Calar Alto 3.5 m telescope on March 16, 1999.

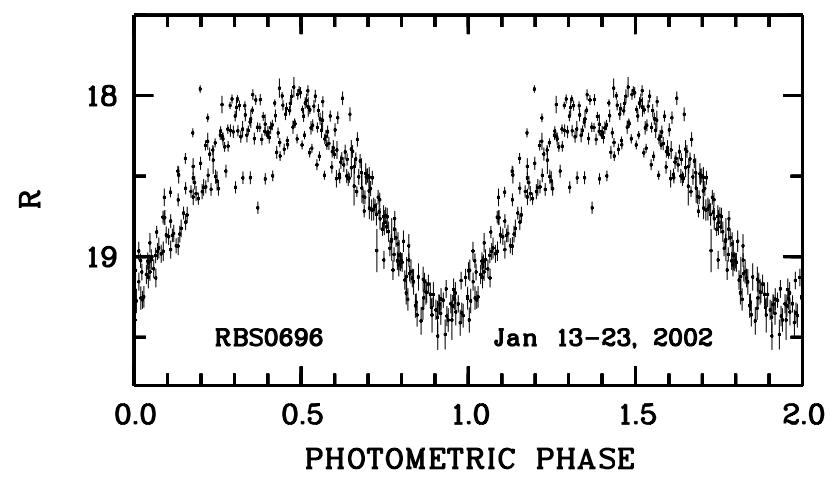

Fig. 10. $R$-band photometry of RBS0696 performed with the Calar Alto $1.23 \mathrm{~m}$ telescope. The data were phase-folded (not averaged) over the period of $99.38 \mathrm{~m}$.

The RASS X-ray spectrum contains 77 photons. It shows the typical shape of an X-ray spectrum of a polar with a hard bremsstrahlung-like tail and a soft blackbody component.

The system shows all the hallmarks of a polar system, an emission line spectrum with asymmetric emission lines, also from high ionization species, a two-component X-ray spectrum and changes between high and low states. The periodic modulation of the optical light curve is interpreted as orbital period. At a period of $82-87$ min the spectral type of the secondary must be later than M6.

The shape of the light curve is similar to that of e.g. V834 Cen, indicating a similar accretion geometry with the main accretion spot being always in the visible hemisphere. The orbital minimum in such a geometry is caused by cyclotron beaming and stream occultation and occurs, when the observer looks almost perpendicular onto the accretion spot. The secondary minimum occurs due to a partial self-eclipse of the accretion spot behind the limb of the white dwarf. The likely orbital inclination is in the range $30^{\circ}-60^{\circ}$, the co-latitude of the accretion spot conversely in the range $60^{\circ}-30^{\circ}$.

\section{4. $R B S 0696(=1 R X S J 082051.2+493433)$}

Source \#696 was identified as cataclysmic variable also by Cao et al. (1999) within their identification programme of sources 


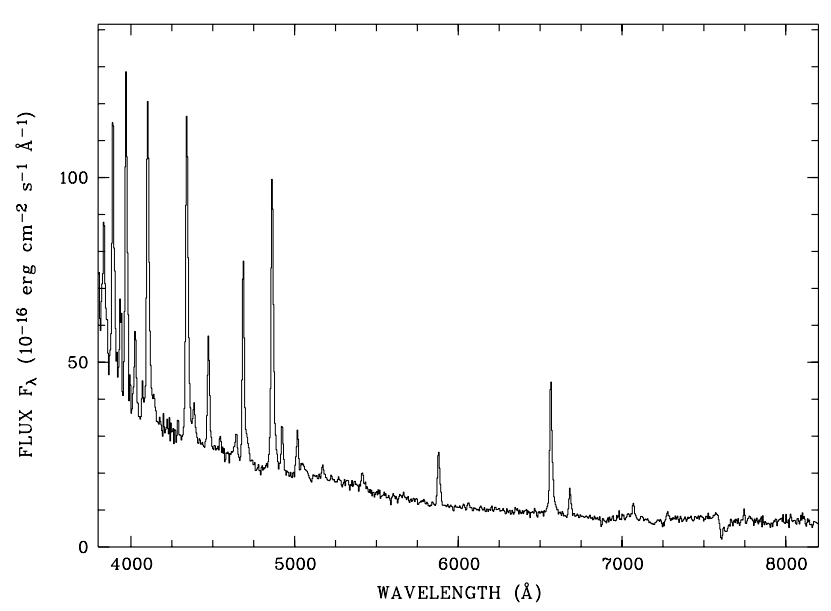

Fig. 11. Discovery spectrum of RBS1563, obtained May 19, 1996, at Zelenchukskaya.

with high ratio between RASS X-ray and optical (DSS) flux. Our identification spectrum of the $V=19.5$ optical counterpart, a $15 \mathrm{~min}$ exposure taken with the $3.5 \mathrm{~m}$ telescope at Calar Alto, Spain, equipped with the MOSCA instrument on March 16, 1999, is reproduced in Fig. 9. It shows H, HeI, and HeII emission lines, respectively, which are superposed on a blue continuum. In the spectrum shown by Cao et al. mainly Balmer emission lines can be recognized. The emission lines in our spectrum have a larger equivalent width and the spectrum contains species with higher ionization potential. This is indicative of an accretion rate change. No obvious spectral signature from the secondary star could be found. With the spectral resolution given it is impossible to judge, whether the emission lines are asymmetric or have multiple components.

Photometric observations using the $1.23 \mathrm{~m}$ telescope at Calar Alto were performed during five nights between January 13 at 23, 2002. Differential $R$-band magnitudes were derived with respect to the comparison star U1350_07362511 (USNO A2), which has $R=15.3$. Smooth, quasi-sinusoidal variations between $R=18.0-19.4$ became evident with a period $(99.38 \pm 0.03) \mathrm{min}$. No other period was found and we regard $P=99.38 \mathrm{~min}$ as the orbital period of the binary. The phase-folded data are shown in Fig. 10. It shows a large scatter by $\sim 0.5$ mag at optical maximum and negligible scatter at optical minimum. This property is typical of polars and caused by irregular accretion at the hot spot. The bright phase was centered on HJD 2452287.7318, 2452289.4597, 2452289.5283, 2452289.5979, 2452290.4224, 2452297.6691, 2452297.7409, respectively.

The softness of the X-ray spectrum, the high ionization emission line spectrum, an the presence of only one period, the likely binary period, make the identification as magnetic $\mathrm{CV}$ of AM Herculis type very likely.

\section{5. $R B S 1563(=1 R X S J 161008.0+035222)$}

RBS1563 was initially observed with the SAO 6m telescope at Zelenchukskaya, were the discovery spectrum was taken (Fig. 11). The spectrum shows the typical hallmarks of a magnetic $\mathrm{CV}$, a blue continuum, broad asymmetric emision lines
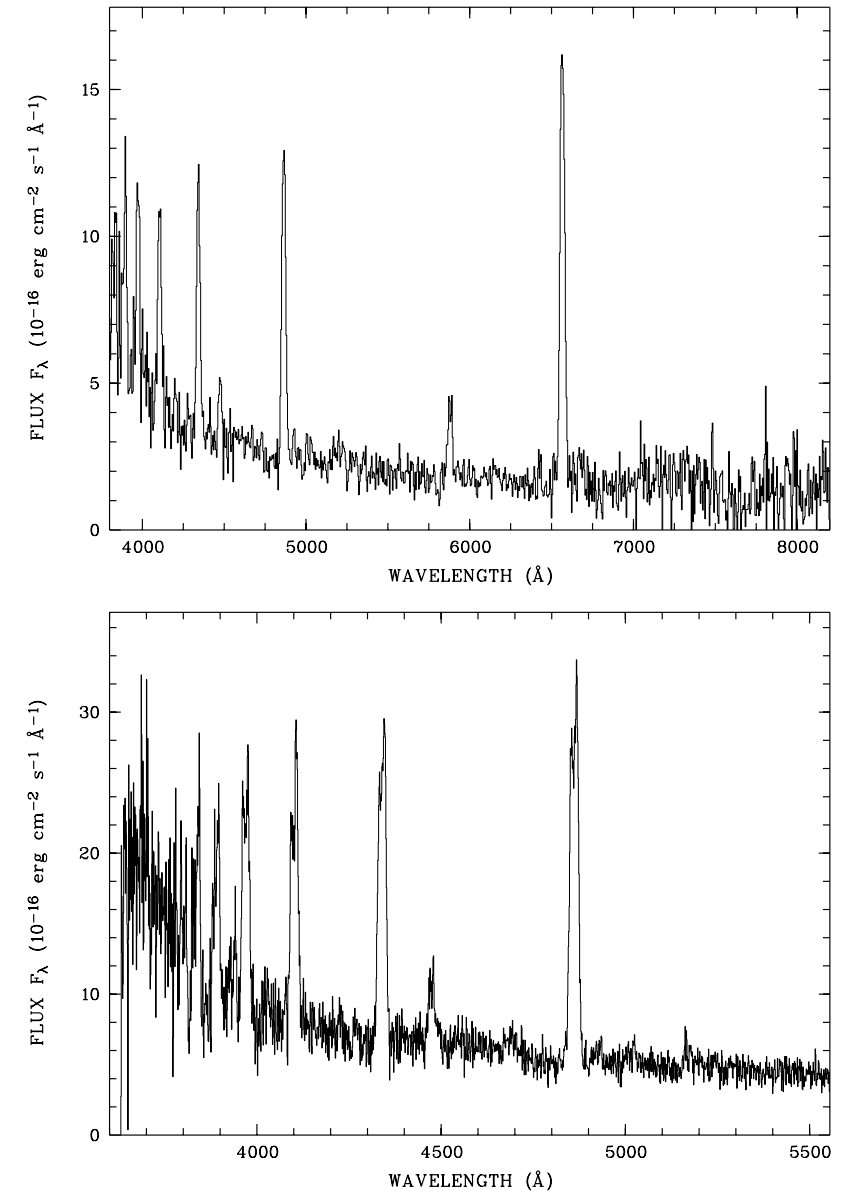

Fig. 12. (top) Discovery spectrum of RBS0372 obtained October 7, 1996, at Zelenchukskaya. (bottom) Spectrum with moderate resolution of RBS0372 obtained August 23, 1998, at SAAO.

of $\mathrm{H}, \mathrm{HeI}$ and HeII, and some indication of the secondary star in the near infrared spectral range. These properties are similar to those reported in the single spectrum shown by Jiang et al. (2000).

During the RASS a total of 207 photons were collected. $\mathrm{X}$-ray variability by $\sim 100 \%$ is clearly present, reaching a peak count rate of about $1.4 \mathrm{cts} / \mathrm{s}$. The resulting spectrum clearly contains two components, a soft blackbody-like component with $k T \simeq 30 \mathrm{eV}$, and a hard bremsstrahlung-like component (fixed at $20 \mathrm{keV}$ for a spectral fit). Although prominent, the soft component was not as dominant as in other magnetic CVs, which lead to a moderate ROSAT hardness ratio.

Follow-up spectroscopy and photometry took place using the ESO/Danish $1.5 \mathrm{~m}$ telescope and the AIP $70 \mathrm{~cm}$ telescope. The rather large observational body thus collected deserves a separate publication (Schwarz et al., in preparation), and we give a very brief summary here.

RBS1563 is a magnetic CV of AM Herculis type, displaying high and low accretion states. The orbital period is $187.7 \mathrm{~min}$. In the high state, the optical light curve is roughly sinusoidal, which is suggestive of a one-pole accretion geometry and a moderate orbital inclination, $i=30^{\circ}-60^{\circ}$. 

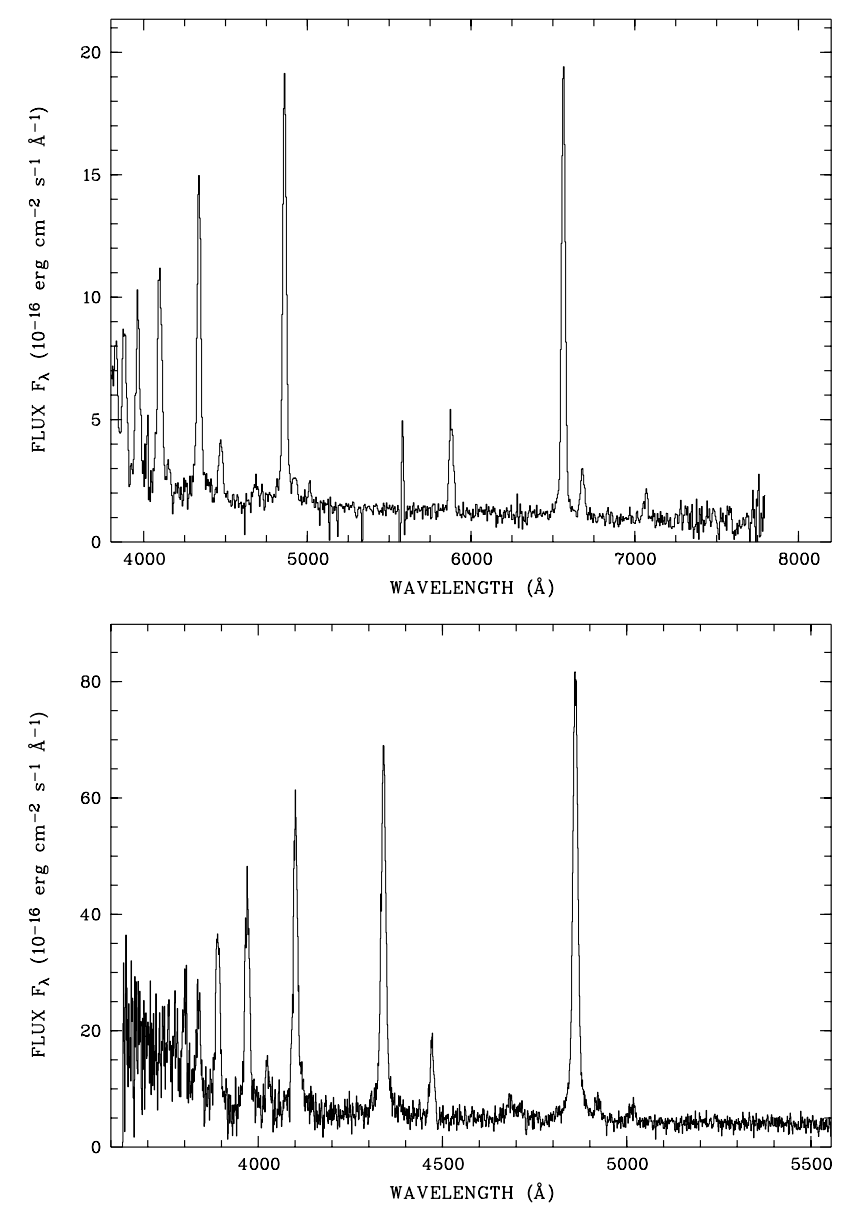

Fig. 13. (top) Discovery spectrum of RBS0490, obtained October 10, 1997, at Zelenchukskaya. (bottom) Mean blue spectrum of RBS0490, obtained on August 23, 1998, at SAAO.

\section{New non-magnetic cataclysmic variables}

\section{1. $R B S 0372$ (= 1 RXS J025538.2-224655)}

The discovery spectrum of RBS0372, obtained on October 7 , 1996, with the SAO 6m-telescope is shown in Fig. 12. The derived below-slit magnitude was $V=18$. 1 . It shows pronounced $\mathrm{H}$-Balmer and neutral HeI emission lines. Emission of HeII was weakly detected. There is no clear indication of the secondary star. Some spectra with higher resolution in the blue and the red spectral range were obtained with the SAAO $1.9 \mathrm{~m}$ telescope. The mean blue spectrum taken on August 23, 1998, with effective exposure of $38 \mathrm{~min}$ is reproduced also in Fig. 12 (bottom panel). It shows the same emission lines as in the discovery spectrum but this time clearly resolved into a double-peaked profile. This is highly reminiscent of emission originating from an accretion disk with rather high inclination. The implied brightness was somewhat higher, $V \sim 17^{\mathrm{m}} 6$, during the SAAO observations. Time-resolved CCD-photometry performed in January 1997 in white light with 10 s time resolution shows pronounced modulation of the optical light curve by up to $0.5 \mathrm{mag}$. Those brightness variations happened on time scales of minutes, hours and nights, but without clear periodicity. The relatively hard X-ray spectrum, the double-peaked emission lines and the rather stochastic intensity variations led

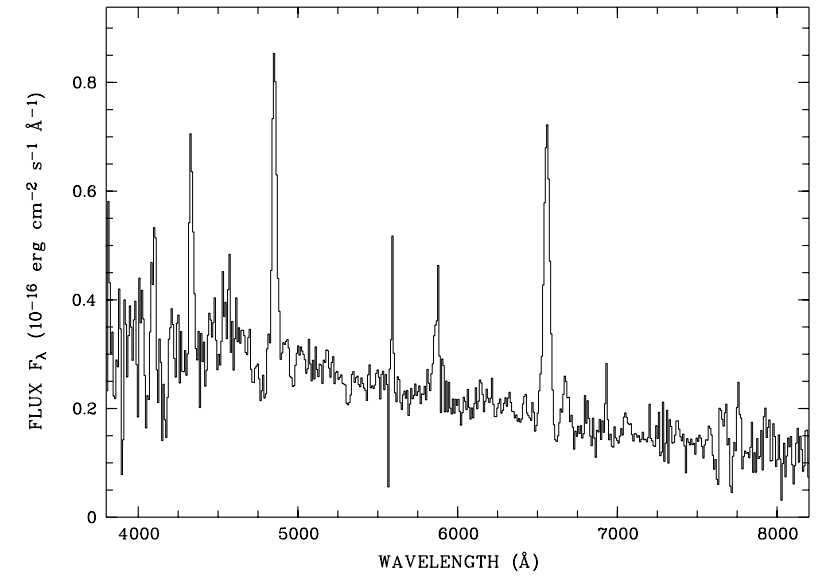

Fig. 14. Discovery spectrum of RBS1411, obtained April 29, 1995, with the ESO/MPG $2.2 \mathrm{~m}$ telescope at La Silla, equipped with EFOSC2.

to our classification as a non-magnetic cataclysmic variable, most probably a dwarf-nova in quiescence.

\section{2. $R B S 0490$ (= $1 R X S J 035410.4-165244)$}

The ROSAT X-ray hardness ratio HR $1=+0.57$ of RBS0490 is the highest among all CVs discussed here. A spectrum constructed from the 87 photons detected in the RASS can be fit with a $20 \mathrm{keV}$ bremsstrahlung component. The identification spectrum of the $V \sim 16^{\mathrm{m}}$ counterpart (Fig. 13, top), obtained October 10, 1997, at Zelenchukskaya, shows pronounced HBalmer and HeI emission lines and weak HeII emission lines. It has a flat, blue continuum. The low-resolution spectrum of \#490 is very similar to that of \#372, although with a somewhat smaller Balmer decrement. Further spectra with moderate resolution were taken on August 23, 1998, at the Sutherland site of SAAO. The mean combined spectrum (four exposures) which has effective integration time of $46 \mathrm{~min}$ is reproduced also in Fig. 13 (bottom). The lines are resolved and double-peaked. The optical and X-ray spectra are typical for a dwarf-nova in quiescence with a moderate to high inclination.

\section{3. $R B S 1411=R H S 40(=1 R X S J 143703.5+234236)$}

RBS1411 was identified already in the initial program aiming at the identification of a bright, hard subsample of the RASS, the ROSAT Hard Survey (RHS). It was listed as number \#40 in the initial RHS-catalog (Fischer et al. 1998). It has a hard RASS X-ray spectrum with HR1 $=0.57$, compatible with a single-component bremsstrahlung spectrum of $k T \simeq 10 \mathrm{keV}$. Further information on \#1411 is sparse. On the DSS the counterpart has an optical brightness of $V=19$. 1 . It was similarly faint on April 29, 1995, when the identification spectrum was taken (Fig. 14). Our spectrum is similar to that shown by Jiang et al. (2000) which confirms our analysis. The spectrum has a blue, smooth continuum with emission lines of hydrogen and neutral helium superposed. The flux decrease towards the blue end of the spectrum is probably not real, but due to an imperfect instrumental response function. Due to the absence of high 


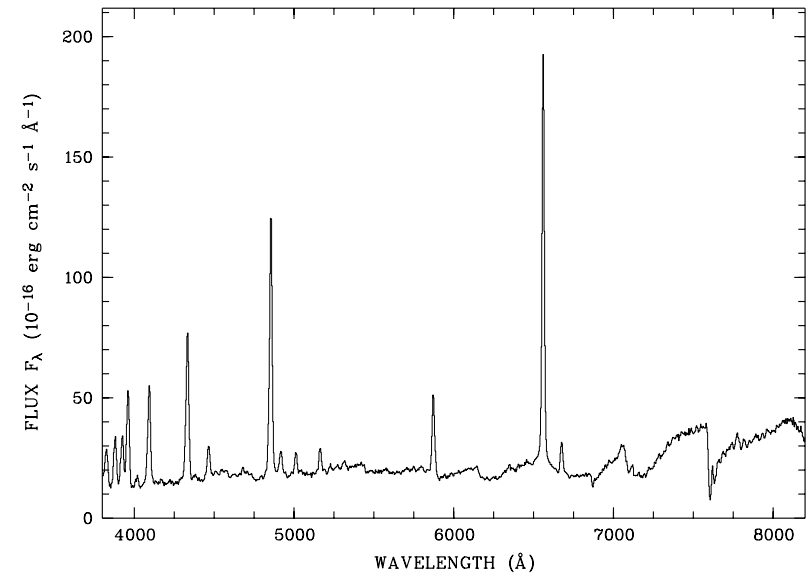

Fig. 15. Discovery spectrum of RBS1955, obtained December 2, 1997, with the $1.5 \mathrm{~m}$ telescope at ESO, La Silla.

ionization species and the hard X-ray spectrum we classify this object as a non-magnetic CV, possibly a dwarf nova in quiescence (at the time of the optical observations). This is supported by the X-ray to optical flux ratio $F_{X} / F_{\text {opt }}=30$ which is found to be unusually high $\left(F_{X}(0.1-2.4 \mathrm{keV})=\right.$ $2.5 \times 10^{-12} \mathrm{erg} \mathrm{cm}^{-2} \mathrm{~s}^{-1}, \quad F_{\text {opt }}=10^{-0.4 V-5.44}=8.3 \times$ $10^{-14} \mathrm{erg} \mathrm{cm}^{-2} \mathrm{~s}^{-1}$ ). This is the highest value among all nonmagnetic CVs in the RBS, at the extreme end of the DN population and two orders of magnitude higher than the nova-like variables (for comparison see the synoptic paper by Beuermann \& Thomas 1993, their Fig. 2).

\section{4. $R B S 1955$ (= $1 R X S J 230949.6+213523)$}

With $V \simeq 15.6$, source \#1955 is by far the brightest of the new interacting binaries at optical wavelengths. The identification spectrum taken with the ESO $1.5 \mathrm{~m}$ telescope at La Silla is reproduced in Fig. 15. A spectrum similar to ours was reported also by Wei et al. (1999). They classified the system as $\mathrm{CV}$ without further specification. The continuum is dominated by emission from an M-type star. Strong emission lines of $\mathrm{H}$ Balmer and HeI are superimposed.

In the RASS $101 \mathrm{X}$-ray photons were collected. X-ray variability is evident on a $2 \sigma$ level. Explanation of the RASS X-ray spectrum requires two radiation components, a soft blackbodylike component with $k T_{\mathrm{bb}}=38 \mathrm{eV}$, and a hard bremsstrahlunglike component which we fixed at $20 \mathrm{keV}$.

Optical variability with an amplitude of $\sim 0.3 \mathrm{mag}$ was detected in differential $V$-band photometry, performed with the $70 \mathrm{~cm}$ telescope of the AIP at two occasions in 1998 (see Fig. 16 for an example). A sequence of three spectra taken in the night of discovery with a time resolution of $10 \mathrm{~min}$ does not show any variablity in the emission line pattern, neither in flux nor in position.

A spectral classification using the schemes by Kirkpatrick et al. (1999) and Martín et al. (1999) revealed a spectral type of M3.

On the basis of the optical spectrum with its strong Mtype stellar component, we tentatively classified this object as symbiotic binary (Schwope et al. 2000). The possible shorttimescale variability does not contradict this classification (see

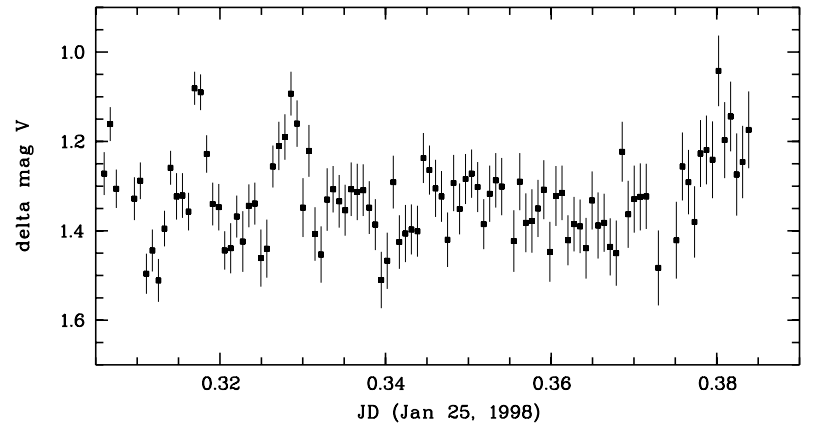

Fig. 16. CCD-photometry of RBS1955 obtained January 25, 1998, at the Babelsberg site. Plotted are $V$-band differential magnitudes with respect to the comparison star 26 arcsec WNW to the source.

Sokoloski et al. 2001 for a comprehensive optical variability study of symbiotics). If this classification would be correct, RBS1955 would only the third symbiotic in the RBS, the others being AG Dra and St Ha32, which both display supersoft spectra. \#1955 also has a soft component, but there is a hard component present in addition.

The classification as a symbiotic binary, however, is far from being unique. As U. Munari kindly pointed out to us, the complete absence of high ionization lines, the rather high HeI triplet to singlet ratio 5876/6678 and the rather flat Balmer decrement are not compatible with this tentative classification. The absence of a strong NaI D absorption at $5893 \AA$ seems to exclude a dwarf classification for the $\mathrm{M}$ star (which would suggest a normal CV). On the other hand, there is $\mathrm{CaH}$ absorption at $6382 \AA$, which serves as discriminant between dwarfs and giants and is usually seen in dwarf stars only (Kirkpatrick et al. 1991; Torres-Dodgen \& Weaver 1993). We therefore cannot exclude a nature as e.g. a dwarf nova in quiescence.

If the secondary should be a Roche-lobe filling dwarf star of spectral type M3, then the distance to RBS1955 would only be $\sim 30 \mathrm{pc}$.

Its brightness, unknown nature and the likely variability of the source make it a prime target for detailed follow-up, with monitoring observations in the optical in order to search for possible outbursts, but particular in the UV/X-ray range in order to search for a hot primary component.

SIMBAD lists RBS1955 as the emission line galaxy UCM $2307+2118$ (Zamorano et al. 1996). The coordinates in the Zamorano paper for the galaxy and for RBS1955 are identical. The classification of RBS1955 as a CV is undoubted, we guess, that the object was misclassified in the earlier publication.

\subsection{RBS1969 (= 1RXS J231532.3-304855)}

Source \#1969 received a very short exposure during the RASS, which allowed to collect 21 source photons only. The derived $\mathrm{X}$-ray spectrum is compatible with a hard bremsstrahlung component. The discovery spectrum, a 15 min exposure shown in Fig. 17, was obtained on November 30, 1997, with the ESO $1.5 \mathrm{~m}$ telescope. Further medium-resolution spectroscopy was obtained from SAAO on August 23, 1998. All spectra show emission lines of H-Balmer, HeI and weak HeII together with a Balmer continuum in emission. The emission lines are 

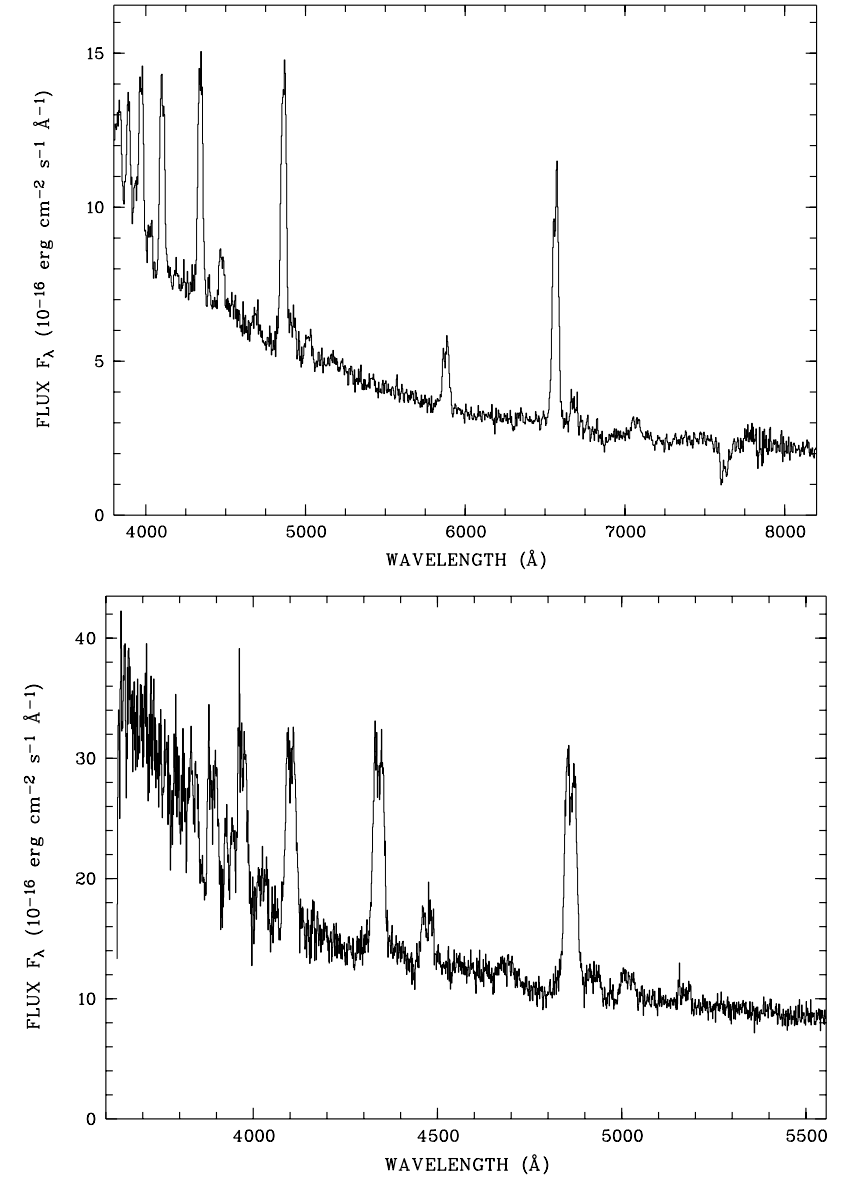

Fig. 17. (top) Identification spectrum of RBS1969, obtained December 2, 1997, with the ESO $1.5 \mathrm{~m}$ telescope. (bottom) Mean blue spectrum of RBS1969, obtained August 23, 1998, with the SAAO $1.9 \mathrm{~m}$ telescope.

double-peaked, which is a typical feature of a highly-inclined accretion disk. The rather hard X-ray spectrum together with the double-peaked emission lines suggest, that RBS1969 is a dwarf nova observed in quiescence at the two epochs. Further photometry and spectroscopy are needed in order to determine the orbital period and to search for a possible eclipse of the disk and the white dwarf, which would make the object a prime target for detailed follow-up.

\section{On the space density of non-magnetic CVs}

In this section we make an attempt to update the space density of cataclysmic variables based on the RBS. We restrict ourselves to the non-magnetic systems. Although the magnetic systems are more numerous, the RBS catalogue lists 24 polars and 5 intermediate polars, they have in general very uncertain distances. Of the 24 polars, about 10 have known or estimated distances, further 4 have lower limits based on the non-detection of secondary star features, and further 10 have no distance estimate at all. A estimate of the space density therefore requires more thorough work on the distance determination of the individual systems, which is beyond the scope of this paper.

Following Patterson (1984) we estimate the absolute brightness $M_{V}$ of the accretion disk in the new non-magnetic
CVs from the observed equivalent width, $E W(\mathrm{H} \beta)$, of the $\mathrm{H} \beta$ line:

$E W(\mathrm{H} \beta)=0.3 M_{V}^{2}+\mathrm{e}^{0.55\left(M_{V}-4\right)}$.

Table 3 lists the relevant numbers for the new CVs. Although RBS0002 = EC 23593-6724 was not originally discovered by us a spectrum was taken in the RBS-program. This is used here to estimate $E W(\mathrm{H} \beta)$ and the distance.

Assuming that the optical emission is dominated by the disk we estimate the distance $d$ to the $\mathrm{CV}$ from the observed apparent and the derived absolute magnitudes. We ignore interstellar absorption which is justified by the rather small distances.

Fluxes in the X-ray band were derived from the measured RASS count rate using PIMMS and a $10 \mathrm{keV}$ thermal bremsstrahlung model with moderate absorption of $N_{\mathrm{H}}=$ $5 \times 10^{20} \mathrm{~cm}^{-2}$. In order to minimize the effect of interstellar absorption, one should better use for flux conversion the count rate in the hard ROSAT band only, $\mathrm{CRH}=0.5-2.0 \mathrm{keV}$, However, the limiting count rate and limiting flux of our survey then would not be properly defined. For the analysis of the space density (see below and Table 4) we therefore stick to the count rates in the full ROSAT band. For better comparison with the Einstein-IPC we use the count rate in the hard band $\mathrm{CRH}$. Conversion factors from count rates to fluxes in the ROSAT and Einstein-IPC bands (0.1-2.4, $0.2-4.0 \mathrm{keV}$ ), are $1.4 \times 10^{-11} \mathrm{erg} \mathrm{cm}^{-2} \mathrm{~s}^{-1}$ (PSPC CR cps) ${ }^{-1}$ and $2.3 \times 10^{-11} \mathrm{erg} \mathrm{cm}^{-2} \mathrm{~s}^{-1}$ (PSPC CRH cps) ${ }^{-1}$, respectively.

For comparison with $\mathrm{CVs}$ discovered in the Einstein galactic plane survey (Hertz et al. 1990), we list in Table 3 the Xray fluxes in the Einstein band. Optical fluxes are derived using $\log F_{\text {opt }}=-0.4 V-5.44$. The optical and X-ray data used for the flux calculation were not taken simultaneously, hence the flux ratios might be incorrect or misleading. Taken the data in Table 3 as face values, we can compare the new RBS-CVs with those found by Hertz et al. (1990) in the Einstein galactic plane survey (GPX, their Fig. 9). Similar to their new GPX CVs the new RBS-CVs have a large $\mathrm{H} \beta$ equivalent widths and a high $F_{\mathrm{X}} / F_{\text {opt }}$-ratio. Patterson \& Raymond (1985) derived an empirical relation between the equivalent widths $E W(\mathrm{H} \beta)$ and the ratio $F_{\mathrm{X}} / F_{\text {opt }}$, which predicts the value of $F_{\mathrm{X}} / F_{\text {opt }}$ to within a factor of 3 . The prediction due to their relation is listed in the second to last column of Table 3. It agrees reasonably well with the observed values of \#372 and \#1969, marginally with \#490, and it disagrees with \#0002 and \#1411. Both systems were found to be much brighter at X-ray wavelengths (or fainter at optical wavelengths) than predicted by the empirical formula. The deviations might be related to an outburst at the time of the RASS or might hint to a somewhat peculiar nature of the sources. The luminosities in the IPC-band range from $\log L_{(0.2-4.0)}=29.5-32.1$, i.e. extending the normal range of CVs to fainter luminosities (compare with e.g. Patterson \& Raymond 1985, their Fig. 5).

Similarly to Hertz et al. (1990) we estimate the space density of the non-magnetic CVs in the RBS by a $1 / V_{\max }$ method. Since our CVs have high galactic latitude and some of them are at a distance in excess of the likely scale height of CVs, which we assume to be $h=200 \mathrm{pc}$, we use the modified method by 
Table 3. Optical and X-ray data of new non-magnetic CVs.

\begin{tabular}{rccrcccccc}
\hline \hline RBS\# & $\begin{array}{c}E W(\mathrm{H} \beta) \\
{[\AA]}\end{array}$ & $\begin{array}{c}M_{V} \\
{[\mathrm{mag}]}\end{array}$ & $\begin{array}{r}D \\
{[\mathrm{pc}]}\end{array}$ & $\begin{array}{c}\mathrm{CRH} \\
{\left[\mathrm{s}^{-1}\right]}\end{array}$ & $\log F_{0.2-4.0}$ & $\log F_{\mathrm{opt}}$ & $\log F_{0.2-4.0} / F_{\mathrm{opt}}$ & $\left(\log F_{0.2-4.0} / F_{\mathrm{opt}}\right)_{P R 85}^{(c)}$ & $\begin{array}{c}L_{0.2-4.0} \\
{\left[\mathrm{erg} \mathrm{s}^{-1}\right]}\end{array}$ \\
\hline 2 & $3^{(a)}$ & 6 & 500 & 0.20 & -11.34 & -11.16 & -0.18 & -1.52 & 32.1 \\
372 & 140 & 12.3 & 130 & 0.10 & -11.63 & -12.55 & 0.92 & 0.90 & 30.7 \\
490 & 230 & 13.4 & 33 & 0.18 & -11.38 & -11.84 & 0.46 & 1.21 & 29.7 \\
1411 & 75 & 10.8 & 460 & 0.20 & -11.34 & -13.08 & 1.74 & -51 & 32.1 \\
1955 & - & - & $30^{(b)}$ & 0.13 & -11.53 & - & - & - & 29.5 \\
1969 & 70 & 10.5 & 165 & 0.18 & -11.38 & -12.08 & 0.70 & 0.47 & 31.1 \\
\hline
\end{tabular}

(a) Pattersons relation is invalid for very small EW, we used $\mathrm{EW}=6$ for an estimate of $M_{V}$.

(b) Distance derived assuming ZAMS secondary.

(c) Estimate based on a relation by Patterson \& Raymond (1985).

Table 4. Derived quantities of the non-magnetic RBS-CVs. The luminosity in this table is the luminosity in the ROSAT spectral band $0.1-2.4 \mathrm{keV}$.

\begin{tabular}{|c|c|c|c|c|c|c|}
\hline RBS\# & Name & $\left.\begin{array}{c}D \\
{[\mathrm{pc}]}\end{array}\right]$ & $\begin{array}{c}\log L_{X} \\
{\left[\mathrm{~s}^{-1}\right]} \\
\end{array}$ & $\begin{array}{c}V_{\text {act }} \\
{\left[\mathrm{pc}^{3}\right]}\end{array}$ & $\begin{array}{c}V_{\text {gen }} \\
{\left[\mathrm{pc}^{3}\right]}\end{array}$ & $V_{\text {act }} / V_{\text {gen }}$ \\
\hline 2 & EC 23593-6724 & $500:^{r 1}$ & 32.0 & $5.5 e+07$ & $6.3 e+07$ & +0.87 \\
\hline 22 & WW Cet & $100^{r 2}$ & 31.0 & $1.5 e+06$ & $6.7 e+06$ & +0.22 \\
\hline 280 & TT ARI & $135^{r 3}$ & 31.1 & $3.8 \mathrm{e}+06$ & $9.3 e+06$ & +0.40 \\
\hline 288 & WX HYI & $265^{r 4}$ & 31.6 & $2.2 \mathrm{e}+07$ & $4.1 \mathrm{e}+07$ & +0.55 \\
\hline 372 & & $130^{r 1}$ & 30.8 & $3.5 e+06$ & $4.3 e+06$ & +0.83 \\
\hline 490 & & $33^{r 1}$ & 29.6 & $6.8 \mathrm{e}+04$ & $8.3 e+04$ & +0.82 \\
\hline 512 & VW Hyi & $65^{r 4}$ & 30.9 & $4.7 e+05$ & $4.7 e+06$ & +0.10 \\
\hline 694 & SU UMa & $280^{r 4}$ & 32.1 & $2.0 \mathrm{e}+07$ & $8.3 e+07$ & +0.24 \\
\hline 710 & SW UMa & $140^{r 4}$ & 30.9 & $3.5 e+06$ & $4.7 e+06$ & +0.76 \\
\hline 713 & EI UMa & - & - & - & - & - \\
\hline 728 & BZ UMa & $110^{r 5}$ & 30.9 & $1.9 \mathrm{e}+06$ & $4.6 e+06$ & +0.40 \\
\hline 1008 & T Leo & $100^{r 6}$ & 31.1 & $1.6 e+06$ & $7.7 e+06$ & +0.21 \\
\hline 1411 & RHS40 & $460^{r 1}$ & 32.0 & $7.1 \mathrm{e}+07$ & $8.8 \mathrm{e}+07$ & +0.81 \\
\hline 1900 & TY PsA & $300:^{.1}$ & 31.7 & $2.3 e+07$ & $3.6 e+07$ & +0.64 \\
\hline 1955 & & $30^{r 1}$ & 29.5 & $5.3 e+04$ & $5.7 e+04$ & +0.93 \\
\hline 1969 & & $165^{r 1}$ & 31.1 & $6.5 e+06$ & $1.0 \mathrm{e}+07$ & +0.65 \\
\hline
\end{tabular}

${ }^{r 1}$ this work, ${ }^{r 2}$ Verbunt et al. (1997), ${ }^{r 3}$ Patterson (1984), ${ }^{r 4}$ Warner (1987), ${ }^{r 5}$ Ringwald et al. (1994), ${ }^{r 6}$ Sproats et al. (1996).

Tinney et al. (1993). Their method of calculation of a generic volume, $V_{\text {gen }}$, accounts for an exponential density distribution $\rho \propto \exp [(-d \sin b) / h]\left(d\right.$ : distance, $b$ : galactic latitude). $V_{\text {gen }}$ is calculated by

$V_{\text {gen }}=\Omega \frac{h^{3}}{\sin ^{3} b}\left(2-\left(\xi^{2}+2 \xi+2\right) \mathrm{e}^{-\xi}\right)$

with $\xi=d \sin b / h$ and $\Omega$ the solid angle of the survey ( $2 \pi$ in our case). The maximum generic volume $V_{\text {gen }}$ is computed using this formula with the maximum possible distance of the particular source which would allow its detection at the flux limit of the survey. One particular $\mathrm{CV}$ then contributes $1 / V_{\text {gen }}$ to the space density $\rho_{X}$, i.e. the space density is $\rho_{X}=\sum \frac{1}{V_{\text {gen }}}$. The corresponding numbers for $V_{\text {act }}$ and $V_{\text {gen }}$ are listed in Table 4 . $V_{\text {act }}$ is the volume covered by this particular source at the measured distance according to Eq. (2).

Using these, the space density of the X-ray selected, nonmagnetic, high-galactic latitude $\mathrm{CVs}$ is $\rho_{X} \geq 3.1 \times 10^{-5} \mathrm{pc}^{-3}$. RBS0713 (= EI UMa) is missing in the sum due to its unknown distance. The two new nearby, low-luminosity CVs \#490 and
\#1955 have the highest weight in the sum, but both have a somewhat uncertain distance. Should their distance be much greater than the $\sim 30 \mathrm{pc}$ derived here, the lower limit of the space density decreases. When omiting these two sources completely, the density becomes $\rho_{X}=1.5 \times 10^{-6} \mathrm{pc}^{-3}$.

$V_{\text {act }} / V_{\text {gen }}$ has a uniform distribution between 0 and 1 , the mean value is 0.56 for the 15 systems with measured or estimated distances, the uniformity is somewhat higher, if \#490 and \#1955 are omitted. The mean value then is 0.51 .

Our limit of the space density is in good agreement with the recent estimate by Patterson (1998), who derives a density of $\sim 10^{-5} \mathrm{pc}^{-3}$. The reader is refered to his paper, where a thorough discussion of the possible selection effects in various surveys is given.

\section{Discussion and conclusions}

We have presented an observational summary of new cataclysmic variables identified in the RBS. Among the 11 new RBS-CVs are 6 polars, 4 dwarf nova candiates and one 


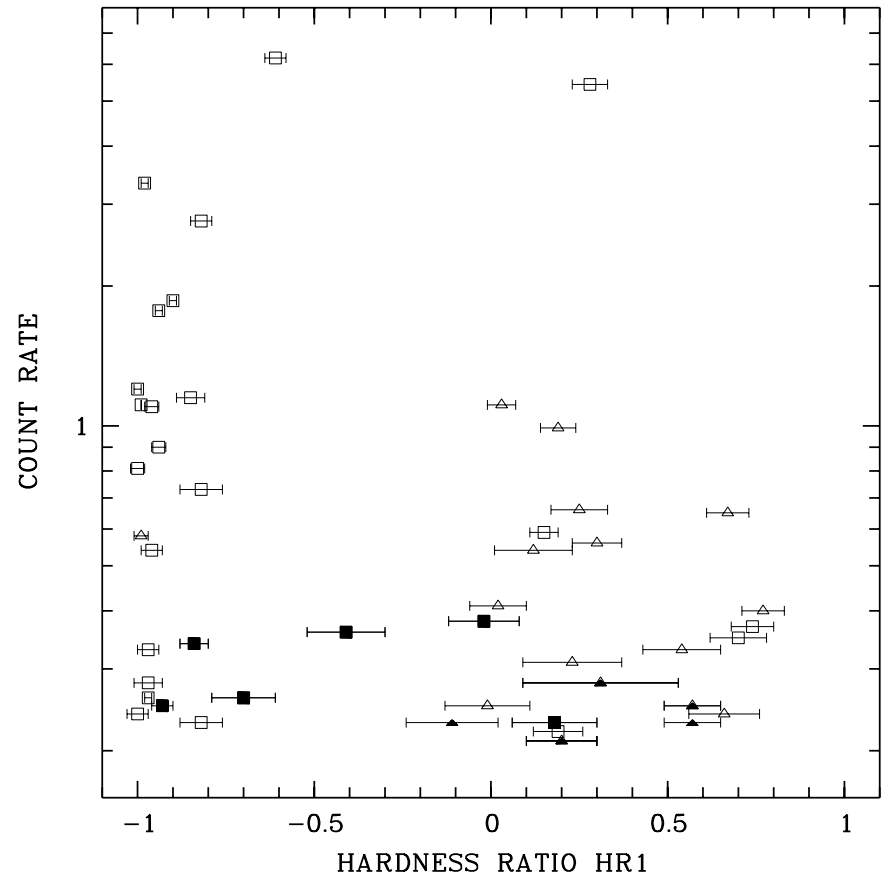

Fig. 18. X-ray colour magnitude diagram of all RBS-CVs. Squares indicate magnetic systems (polars or intermediate polars), triangles indicate non-magnetic systems (dwarf novae, nova-likes and symbiotics). Filled symbols refer to systems discussed in this paper, open symbols to the other CVs in the RASS.

system with uncertain classification, which was tentatively identified as symbiotic binary but could also be a dwarf nova. Orbital periods could be determined for all polars, they range from $87.1 \mathrm{~min}$ to $187.7 \mathrm{~min}$. One of the new polars was found to be asynchronous (RBS1735, Schwope et al. 1997), another displays a highly peculiar cyclotron spectrum (RBS0206, Schwope et al. 1999 and this work). Only RBS0206 has a measured field strength, the other systems do not show cyclotron or Zeeman lines.

Figure 18, a X-ray colour-magnitude diagram, puts the new systems in the context of all CVs in the RBS. The RBS lists $46 \mathrm{CVs}$ and 1 symbiotic. Note that these are systems at highgalactic latutide above $|b|>30^{\circ}$ only. Of the $45 \mathrm{CVs} 29$ are magnetic (polars and intermediate polars) and 16 non-magnetic (DN, nova-like). The Figure shows the known dichotomy between magnetic and non-magnetic systems which is explained in terms of the typical X-ray spectra of the various sub-classes (Beuermann \& Thomas 1993). Polars often have X-ray spectra dominated by soft X-ray, blackbody-like emission. Three of the new polars (\#206, \#324, and \#696) follow the normal pattern, while further three have rather hard X-ray spectra (\#541, $\# 1563$, \#1735). The reason for this non-conformity is not clear, \#541 and \#1563 appear to be quite normal polars with a onepole accretion geometry. Only \#1735 is unusual, since it belongs to the rare sub-class of slightly asynchronous polars, where the slightly different accretion mode might influence the release of gravitational energy.

The systems described here enlarge the population at the extremes of the X-ray/optical flux plane. Beuermann \&
Thomas (1993) plotted all CVs known at that time in the RASS count-rate/V-magnitude plane with lines of constant $F_{X} / F_{\mathrm{opt}}$ indicated. The new magnetic RBS-CVs are located at the low end of the distribution, while the new non-magnetic systems are located at the X-ray luminous end of the distribution. RBS1411 seems to represent the extreme example, it lies in the domain which is otherwise populated only by the magnetic CVs.

The high $F_{\mathrm{X}} / F_{\text {opt }}$ ratios of the DN (particularly \#1411) might be explained by possible outbursts in these systems during the RASS observation. The spectra of the dwarf nova candidates show no broad absorption features of the primary star, and these systems are unlikely short period CVs of the WZ Sge subclass.

No new obvious IP candidate was found, which indicates that our census of these intrinsically bright systems is already complete at high galactic latitudes.

We have tentatively identified two new nearby, lowluminosity CVs (\#490 and \#1955). Whether there is a large population of these sources as predicted by Hertz et al. (1990) is difficult to answer conclusively but our sources might be the first representatives of this population.

We have discussed the observational properties of the new cataclysmic variables found in the RASS. We could determine orbital periods for all magnetic CVs, period information for the non-magnetic $\mathrm{CVs}$ is lacking. The prospects of finding the periods by photometry is good for \#372, \#490, and \#1969, since the double-peaked emission lines indicate a moderate to high inclination. The two latter systems might show eclipses, the light curves already obtained for \#372 do not show obvious signs of eclipses.

Acknowledgements. We acknowledge helpful comments by Dr. U. Munari (Padova) and N. Lodieu (AIP) on the spectrum of RBS1955. We thank our referee, Dr. D. Christian, for his comments, which helped to improve the paper. This project was supported by the DLR through grant 50 OR 97068.

The ROSAT project is supported by the Bundesministerium für Bildung, Wissenschaft, Forschung und Technologie (BMBF/DLR) and the Max-Planck-Gesellschaft. We thank the ROSAT team for performing the All-Sky Survey and producing the RASS Bright Source Catalogue.

This research has made use of the SIMBAD database operated at CDS, Strasbourg, France, and the NASA/IPAC Extragalactic database (NED) operated by the Jet Propulsion Laboratory, California Institute of Technology under contract with the National Aeronautics and Space Administration. Identification of the RASS X-ray sources was greatly facilitated by use of the finding charts based upon the COSMOS scans of the ESO/SERC J plates performed at the Royal Observatory Edinburgh and APM catalogue based on scans of the red and blue POSS plates performed at the Institute of Astronomy, Cambridge, UK.

Based in part on photographic data of the National Geographic Society - Palomar Observatory Sky Survey (NGS-POSS) obtained using the Oschin Telescope on Palomar Mountain. The NGS-POSS was funded by a grant from the National Geographic Society to the California Institute of Technology. The plates were processed into the present compressed digital form with their permission. The Digitized Sky Survey was produced at the Space Telescope Science Institute under US Government grant NAG W-2166. 


\section{Appendix A: Finding charts of the new CVs}

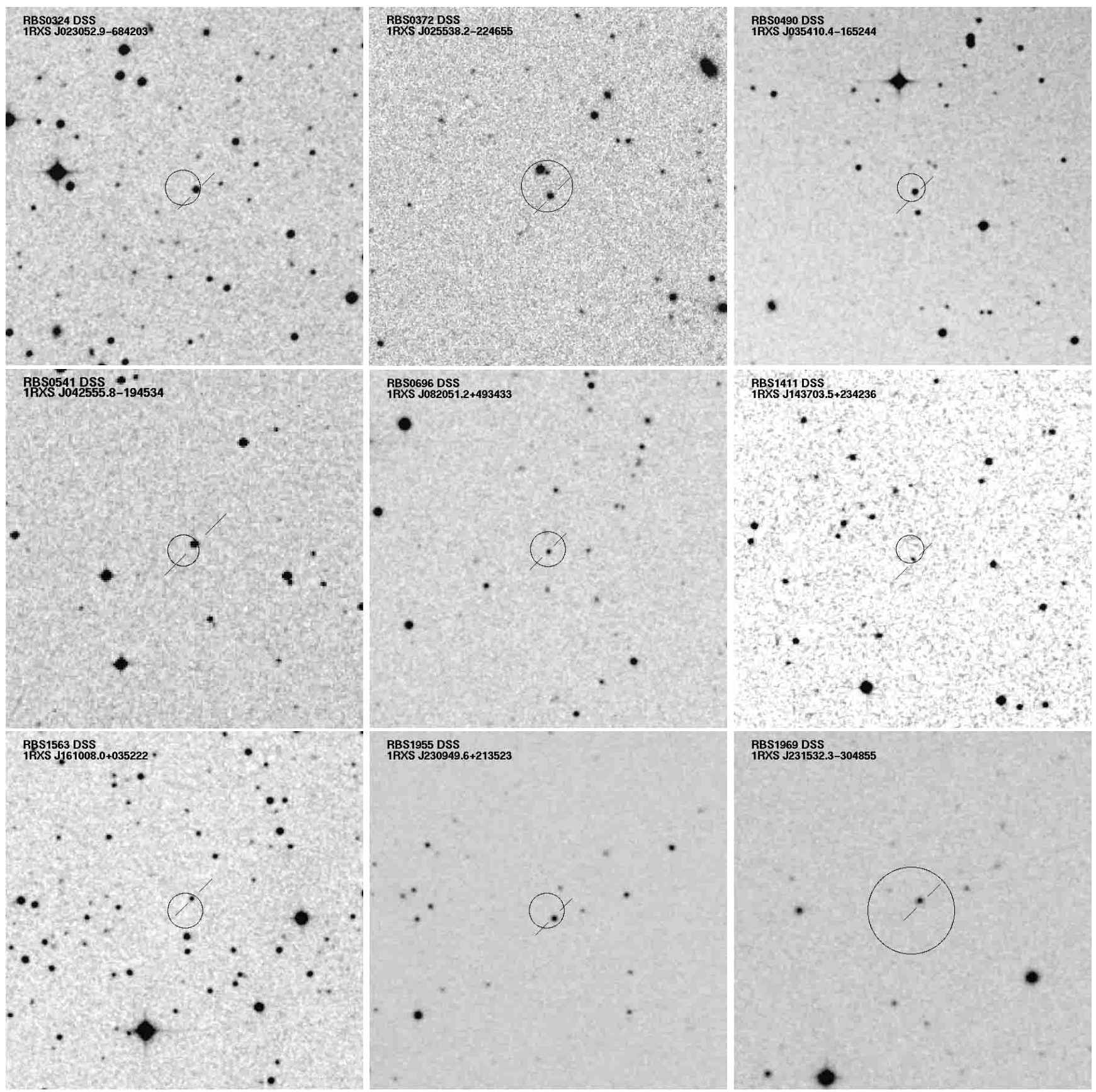

Fig. A.1. Finding charts of the objects presented in this paper and not published elsewhere. Each chart has a size of $5 \times 5$ arcmin with North at top and East to the left. The CVs are marked.

Note added in proofs: The nature of RBS1969 as dwarf nova was independently confirmed by Kato et al. 2001 (IBVS 5023), who discovered dwarf nova outbursts with an estimated recurrence time of $\sim 110$ days and a brightness range of $m_{\mathrm{V}}=13.4$ 17.3.

In the proof reading stage of our accepted paper (astro-ph/0210059) Dr. J. Thorstensen made us aware of possible proper motions of the two candidate nearby dwarf novae RBS0490 and RBS1955. Kato \& Hitoshi report in the IBVS (astro-ph/0211132) a proper motion of 0.'069 $\pm 0 .{ }^{\prime} 012$ for RBS1955 based on Schmidt plates obtained at two epochs. With the help of Drs. R.-D. Scholz and W. Dehnen, both AIP, we searched the databases for position measurements of the two stars. We found data from 12 epochs for RBS0490, spanning the interval 1955.952 - 1999.861, and from 4 epochs for RBS1955 (1951.611-1991.754). For \#490 we derive a proper motion of $\mu_{x}=\mu_{\alpha} \cos \delta=-8 \pm 5 \mathrm{mas} \mathrm{yr}^{-1}, \mu_{y}=\mu_{\delta}=$ $-110 \pm 6 \mathrm{mas} \mathrm{yr}^{-1}$, for \#1955 we derive $\mu_{x}=\mu_{\alpha} \cos \delta=$ $-46 \pm 11 \mathrm{mas} \mathrm{yr}^{-1}, \mu_{y}=\mu_{\delta}=-52 \pm 6 \mathrm{mas} \mathrm{yr}^{-1}$. If we assume, that both objects belong to an old disk population with 
$\sigma_{r} \simeq 40 \mathrm{~km} \mathrm{~s}^{-1}$ and a Schwarzschild distribution function, one derives for the given position and proper motion a distance of $31 \pm 63 \mathrm{~km} \mathrm{~s}^{-1}$ and $24 \pm 112 \mathrm{~km} \mathrm{~s}^{-1}$ for RBS0490 and RBS1955, respectively. Although these estimates are not very constraining they support the spectroscopic parallax made in the paper. They exclude a giant nature of the secondary in RBS1955.

Photometry of RBS1955 through an I-filter was obtained during three nights in October 2002 using the Calar Alto $1.23 \mathrm{~m}$ telescope with CCD camera. Variability of up to $0.25 \mathrm{mag}$ was evident. A period search with the analysis-of-variance technique revealed possible periods at 3.8, 4.5, and 5.6 hours. Longer periods cannot be excluded. The shortest periods are roughly compatible with a Roche-lobe filling M3 dwarf. Detailed photometric and spectroscopic follow-up is necessary in order to determine the true nature of this interesting source.

\section{References}

Beuermann, K., \& Schwope, A. D. 1994, in Interacting binary stars, ed. A. W. Shafter, San Francisco, Calif. Astronomical Society of the Pacific, 56, 119

Beuermann, K., \& Thomas, H.-C. 1993, Adv. Sp. Res., 13(12), 115

Beuermann, K., Thomas, H.-C., Reinsch, K., et al. 1999, A\&A, 347, 47

Cao, L., Wei, J.-Y., \& Hu, J.-Y. 1999, A\&AS, 135, 243

Fischer, J.-U., Hasinger, G., Schwope, A. D., et al. 1998, AN, 319, 347

Gänsicke, B., Beuermann, K., \& de Martino, D. 1995, A\&A, 303, 127

Hertz, P., Bailyn, C. D., Grindlay, J. E., et al. 1990, ApJ, 364, 251

van der Heyden, K. 2000, Ph.D. Thesis, Univ. Cape Town

Jiang, X. J., Engels, D., Wei, J. Y., Tesch, F., \& Hu, J. Y. 2000, A\&A, 362,263

Kirkpatrick, J. D., Henry, T. J., \& McCarthy, Jr., D. 1991, ApJS, 77, 417

Kirkpatrick, J. D., Reid, I. N., Liebert, J., et al. 1999, ApJ, 519, 802 de Kool, M. 1992, A\&A, 261, 188

Liebert, J., Stockman, H. S., Williams, R. E., et al. 1982, ApJ, 256, 594

Martín, E. L., Delfosse, X., Basri, G., et al. 1999, AJ, 118, 2466

Mateo, M., \& Schechter, P. 1989, in 1st ESO/ST-ECF Data Analysis Workshop, ed. P. J. Grosbol, F. Murtagh, \& R. H. Warmels, 69

Miyaji, T., Hasinger, G., \& Schmidt, M. 2001, A\&A, 369, 49

Östreicher, R., Seifert, W., Wunner, G., \& Ruder, H. 1990, ApJ, 350, 324

Paerels, F., Heise, J., \& v. Teeseling, A. 1994, ApJ, 426, 313

Patterson, J. 1984, ApJS, 54, 443

Patterson, J. 1998, PASP, 110, 1132

Patterson, J., \& Raymond, J. C. 1985, ApJ, 292, 535

Politano, M. 1996, ApJ, 465, 338

Pye, J. P., McGale, P. A., Allan, D. J., et al. 1995, MNRAS, 274, 1165

Ringwald, F., Thorstensen, J. R., \& Hamwey, R. M. 1994, MNRAS, 271,323

Schwope, A. D. 1990, Rev. Mod. Astron., 3, 44

Schwope, A. D. 1991, Ph.D. Thesis, TU Berlin

Schwope, A. D., Hasinger, G., Lehmann, I., et al. 2000, AN, 321, 1

Schwope, A. D., Schwarz, R., \& Greiner, J. 1999, A\&A, 348, 861

Schwope, A. D., Buckley, D. A. H., O’Donoghue, D., et al. 1997, A\&A, 326, 195

Sokoloski, J. L., Bildsten, L., \& Ho, W. C. G. 2001, MNRAS, 326, 553

Sproats, L., Howell, S. B., \& Mason, K. O. 1996, MNRAS, 282, 1211

Thomas, H.-C., Beuermann, K., Reinsch, K., et al. 1998, A\&A, 335, 467

Tinney, C. G., Reid, I. N., \& Mould, J. R. 1993, ApJ, 414, 254

Torres-Dodgen, A. V., \& Weaver, W. B. 1993, AJ, 105, 693

Verbunt, F., Bunk, W. H., Ritter, H., \& Pfeffermann, E. 1997, A\&A, 327, 602

Warner, B. 1987, MNRAS, 227, 23

Wei, J. Y., Xu, D. W., Dong, X. Y., \& Hu, J. Y. 1999, A\&AS, 139, 575

Zamorano, J., Gallego, J., Rego, M., Vitores, A. G., \& Alonso, O. 1996, ApJS, 105, 343 